Aus der Klinik für Anästhesiologie

(Prof. Dr. M. Quintel)

der Medizinischen Fakultät der Universitätsmedizin Göttingen

\title{
Ambulante Schulterarthroskopie durchgeführt in alleiniger Allgemeinanästhesie oder als Kombination mit einem interskalenären Plexusblock
}

\author{
INAUGURAL-DISSERTATION \\ zur Erlangung des Doktorgrades \\ der Medizinischen Fakultät der \\ Georg-August-Universität Göttingen
}

vorgelegt von

Hauke Onko Janssen

aus Göttingen

Göttingen 2015 
I. Berichterstatter: $\quad$ Prof. Dr. med. J. Hinz

II. Berichterstatter/in: $\quad$ PD Dr. med. T. Walde

III. Berichterstatter/in: $\quad$ Prof. Dr. med. M. Oppermann

Tag der mündlichen Prüfung: 08.12.2015 


\section{Abkürzungsverzeichnis}

ACE

ASA

AWMF

BDA

BDC

BIS

BMI

bzw.

ca.

$\mathrm{cm}$

COPD

DGAI

ggf.

h

$\mathrm{Hz}$

ISB

$\mathrm{kg}$

KG

$\mathrm{mA}$

$\mathrm{mg}$

$\min$

$\mathrm{ml}$

$\mathrm{mm}$

$\mathrm{mmHg}$

ms angiotensin converting enzyme

American Society of Anesthesiologists

Arbeitsgemeinschaft der Wissenschaftlichen

Medizinischen Fachgesellschaften e.V.

Berufsverband Deutscher Anästhesisten e.V.

Berufsverband der Deutschen Chirurgen e.V.

Bispectral-Index

body mass index

beziehungsweise

cirka

Zentimeter

chronic obstructive pulmonary disease

Deutsche Gesellschaft für Anästhesiologie und

Intensivmedizin e.V.

gegebenenfalls

Stunde

Hertz $(1 / \mathrm{sec})$

interskalenärer Plexusblock

Kilogramm

Körpergewicht

Milliampere

Milligramm

Minute

Milliliter

Millimeter

Millimeter-Quecksilbersäule

Millisekunde 
N.

$\mathrm{NaCl}$

NRS

NSAR

o.g.

o.ä.

PONV

$\mathrm{sec}$

v.a.

$\mathrm{VN}$

vS.

VOPM

WHO

$\mu \mathrm{g}$ nervus (Nerv)

Natriumchlorid

Numerische Rating-Skala

nicht steroidale Antirheumatika

oben genannt

oder ähnliches

postoperative nausea and vomiting

Sekunde

vor allem

Vollnarkose $=$ Allgemeinanästhesie

versus

Verband für OP - Management e.V.

World Health Organization

Mikrogramm 
1. Einleitung__ 6

1.1 Allgemeinanästhesie bei ambulanten Operationen___ 8

1.2 Grundlagen der peripheren Leitungsanästhesie__ 10

1.3 Prinzip der Nervenstimulation___ 12

1.4 Interskalenäre Plexusanästhesie____ 13

1.5 Narkoseverfahren für arthroskopische Schulteroperationen ___ 16

1.6 Fragestellung__ 19

2. Material und Methoden___ 20

2.1 Interskalenäre Plexusanästhesie___ 21

2.2 Allgemeinanästhesie __ 23

2.3 Auswertung der Narkoseprotokolle___ 25

2.4 Patientenbefragung___ 27

2.5 Statistische Auswertung___ 28

3. Ergebnisse___ 29

3.1 Patientendaten__ 29

3.2 Prozesszeiten _ 29

3.3 Narkosemittelbedarf __ 30

3.4 Interskalenäre Plexusanästhesie___ 31

3.5 Hämodynamik__ 32

3.6 Arterielle Sauerstoffsättigung___ 34

3.7 Patientenbefragung__ 35

4. Diskussion___ 40

4.1 Bewertung der eigenen Ergebnisse___ 40

4.2 Methodenkritik und Fehlermöglichkeiten__ 50

5. Zusammenfassung__ 52

6. Anhang__ 53

7. Literaturverzeichnis__ 58 


\section{Einleitung}

Mit dem Vertrag „Ambulantes Operieren und stationsersetzende Eingriffe im Krankenhaus“ (AOP-Vertrag) zwischen den Spitzenverbänden der gesetzlichen Krankenversicherungen, der Deutschen Krankenhausgesellschaft e.V. und der Kassenärztlichen Bundesvereinigung im Jahr 2006 (Kassenärztliche Bundesvereinigung 2006) nahm der ökonomische Druck und damit der Anteil ambulanter Operationen in den letzten Jahren kontinuierlich zu (Heller et al. 2009). Laut der Gesundheitsberichterstattung des Bundes stieg in Deutschland die Zahl der Krankenhäuser, die ambulant operieren von 1.059 im Jahr 2002 auf 1.264 im Jahr 2010. Die Anzahl der ambulanten Operationen stieg von 576.613 im Jahr 2002 auf 1.854.125 im Jahr 2010 (Statistisches Bundesamt 2010). Ermöglicht wird dies durch die Entwicklung minimal-invasiver chirurgischer Techniken und die Verfügbarkeit von kurz wirksamen Anästhetika (Heller et al. 2009). Trotzdem liegt der Anteil der ambulant durchgeführten Operationen in Deutschland immer noch unter $50 \%$, während in anderen Industrieländern teilweise über $80 \%$ der Operationen ambulant durchgeführt werden (Möllmann 2011).

Dabei bietet die ambulante Chirurgie neben der ökonomische Leistungserbringung weitere Vorteile wie schlanke Organisationsstrukturen, Unabhängigkeit von Bettenverfügbarkeit, keine Trennung von gewohnter häuslicher Umgebung (Kinder, ältere Patienten) und eine niedrige Inzidenz von nosokomialen Infektionen. Entscheidend ist, dass für ambulant durchgeführte Eingriffe dieselben Qualitätsmaßstäbe gelten wie für stationäre Eingriffe, das heißt der Patient darf keinem erhöhten Risiko ausgesetzt sein, wenn der Eingriff ambulant durchgeführt wird (Heller et al. 2009). Das Risiko eines operativen Eingriffs ergibt sich aus dem allgemeinen Narkoserisiko, der Invasivität des Eingriffs mit dem zu erwartendem Blutverlust, der Operationsdauer und dem präoperativen Status des Patienten, also den Vorerkrankungen. Daraus ergeben sich Bedingungen, die bei einer ambulant durchgeführten Operation gegeben sein müssen. So können nur ASA I-III-Patienten ambulant ope 
riert werden, wobei bei ASA III-Patienten eine enge Absprache mit dem Hausarzt oder Facharzt und eine ausgedehntere präoperative Diagnostik erfolgen müssen. Außerdem müssen akute Infekte oder eine Exazerbation einer Grunderkrankung ausgeschlossen sein. In Bezug auf den Eingriff muss die Operationszeit begrenzt sein (unter 3 Stunden), und es darf nur ein minimales Blutungsrisiko vorliegen. Ambulant werden demnach keine offenen Laparotomien, intrathorakalen oder intrakraniellen Eingriffe oder Eingriffe mit absehbarer Beeinträchtigung der Vitalfunktionen oder der Atemwege durchgeführt. Außerdem muss neben der Einwilligung des Patienten eine ausreichende Kooperationsfähigkeit, eine geringe Entfernung zur Unterbringung mit ausreichender Infrastruktur (Lift, Telefon, sanitäre Einrichtung) und die Anwesenheit einer Begleitperson für die nächsten 24 Stunden gewährleistet sein (Heller et al. 2009).

Für die postoperative Überwachung und die Entlassung des Patienten sind sowohl der Chirurg als auch der Anästhesist verantwortlich. Laut Vereinbarung zur Qualitätssicherung ambulantes Operieren des Berufsverbandes Deutscher Anästhesisten, der Deutschen Gesellschaft für Anästhesiologie und Intensivmedizin und des Berufsverbandes der Deutschen Chirurgen müssen bei der Entlassung des Patienten folgende Kriterien erfüllt sein (DGAI 2006):

- Patient ist im Vollbesitz seiner Schutzreflexe

- stabile Kreislaufverhältnisse

- keine respiratorischen Einschränkungen

- Orientierung nach Zeit und Ort

- kein akutes postanästhesiologisches Erbrechen

- Nahrungsaufnahme möglich

- Sicherstellung einer adäquaten postoperativen Schmerztherapie

Für ambulant durchgeführte Regionalanästhesie-Verfahren gilt zudem:

- Die Blockade von Sensorik und Motorik muss rückläufig sein.

- Bei rückenmarksnahen Verfahren ist zusätzlich die Blasenfunktion zu berücksichtigen. 
Die Zufriedenheit der Patienten mit der Narkose ist vor allem abhängig von postoperativen Schmerzen und postoperativer Übelkeit und Erbrechen. Zu bemerken ist, das bis zu 25\% der Patienten ohne oder mit unzureichender Schmerztherapie nach Hause entlassen werden bzw. bis zu 50\% der Patienten unzureichend über die postoperative Schmerztherapie informiert werden oder die Anweisungen des Arztes nicht verstehen (Möllmann 2011). Obwohl die Vorteile von Lokal- und Regionalanästhesie-Verfahren in Bezug auf die postoperativen Schmerzen bekannt sind und diese Verfahren auch in den S3-Leitlinien der Arbeitsgemeinschaft der Wissenschaftlichen Medizinischen Fachgesellschaften e.V. (AWMF) berücksichtigt sind, werden Lokal- und Regionalanästhesie-Verfahren bei ambulanten Operationen nur sehr selten genutzt (Deutsche Interdisziplinäre Vereinigung für Schmerztherapie 2009). In einer Umfrage von Lux et al. aus dem Jahr 2011 bei niedergelassen Anästhesisten ergab sich, dass 80\% der ambulanten Eingriffe in Allgemeinanästhesie durchgeführt wurden. $12 \%$ der Operationen wurden in Lokalanästhesie, $6 \%$ mittels peripherer Leitungsanästhesie, 2\% mit rückenmarksnahen Verfahren durchgeführt und $9 \%$ in einer Kombination aus Lokalanästhesie und Allgemeinanästhesie (Lux et al. 2011). In 74\% der ambulanten Zentren wird die postoperative Schmerztherapie von den ambulant tätigen Chirurgen übernommen, obwohl mit dem Anästhesisten ein Spezialist vor Ort ist (Lux et al. 2008, Möllmann et al. 2011).

Die besonderen Herausforderungen bei ambulant durchgeführten Eingriffen liegen also neben der Wirtschaftlichkeit und der Patientensicherheit in der Vermeidung der postoperativen Übelkeit und der Sicherstellung einer adäquaten postoperativen Schmerztherapie, da die Patienten noch am Tag der Operation entlassen werden. Die Verwendung von Regionalanästhesieverfahren kann dabei hilfreich sein.

\subsection{Allgemeinanästhesie bei ambulanten Operationen}

Im ambulanten Bereich benötigt man ein Anästhesieverfahren, das gut steuerbar ist und ein schnelles Aufwachen nach der Narkose ermöglicht. Daher wird häufig die 
total intravenöse Anästhesie (TIVA) verwendet. Bei der TIVA werden nur intravenöse Medikamente und keine volatilen Anästhetika verwendet. Eine häufig verwendete Kombination ist die aus Propofol als Hypnotikum mit Remifentanil als Opioid-Analgetikum. Neben der Tatsache, dass kein komplexes Narkosegas-System mit dem nötigen Abgassystem benötigt wird, ermöglicht die total intravenöse Anästhesie ein schnelleres Erwachen und eine bessere postoperative Vigilanz als bei der Verwendung von volatilen Anästhetika. Außerdem können die Patienten bis zu fünf Minuten früher extubiert werden (Grundmann et al. 1998, Juckenhöfel et al. 1999, Gozdemir et al. 2007). Ein weiterer Vorteil der TIVA ist eine niedrigere Inzidenz von postoperativer Übelkeit und Erbrechen (PONV) (Borgeat und Stirnemann 1998, Gupta 2007, Eikaas und Reader 2009, Rowbotham et al. 1998, Hong et al. 2008), eines der häufigsten Probleme im Aufwachraum und ein häufiger Grund für eine verzögerte Verlegung (Heller et al. 2009, Shnaider und Chung, 2006, White und Eng, 2007). Die Inzidenz ist abhängig von Geschlecht, Alter, PONV oder Reisekrankheit in der Anamnese, Raucherstatus und der Narkosedauer (Apfel et al. 1998). Allgemein beträgt das Risiko 20-30\% und kann bei Risikogruppen auch $70-80 \%$ betragen (Gan et al. 2007).

Im Vergleich zu der balancierten Anästhesie, also einer Allgemeinanästhesie mit Verwendung volatiler Anästhetika, gibt es aber auch Nachteile. So ist ein vermehrtes Auftreten von postoperativem Kältezittern (Shivering) beschrieben worden, wenn eine TIVA durchgeführt wurde (Grundmann et al. 1998, Gozdemir et al. 2007). Das Kältezittern führt zu einer Minderung des Wohlbefindens des Patienten, zu einem vermehrten Wundschmerz, und es konnte gezeigt werden, dass durch den erhöhten Sauerstoffverbrauch Patienten mit erniedrigter Koronarreserve gefährdet sind, eine kardiale Ischämie zu erleiden (Frank et al. 1993).

Ein weiteres Problem bei Verwendung der TIVA, vor allem mit kurzwirksamen Opioiden wie Remifentanil oder Alfentanil, können stärkere postoperativ auftretende Schmerzen darstellen und führen damit zu einem früheren und vermehrten Schmerzmittelbedarf nach der Operation (Gozdemir et al. 2007, Hong et al. 2008). 
Ein zusätzliches Problem könnte die opiat-induzierte Hyperalgesie darstellen, die vor allem bei Patienten beobachtet werden kann, die langfristig Opioide zur Schmerztherapie erhalten. Die Relevanz in Bezug auf das akute Auftreten nach einer perioperativen Opioidgabe ist noch nicht abschließend geklärt und ist Gegenstand aktueller Forschung (Lee et al. 2011). Guignard et al. beschrieben aber im Jahr 2000 einen erhöhten Schmerzmittelbedarf bei Patienten, die intraoperativ höhere Dosen von Remifentanil erhalten haben (Guignard et al. 2000).

\subsection{Grundlagen der peripheren Leitungsanästhesie}

Die periphere Leitungsanästhesie ist neben der rückenmarksnahen Leitungsanästhesie (Spinalanästhesie und Epiduralanästhesie) und der intravenösen Leitungsanästhesie ein Verfahren zur Regionalanästhesie. Als periphere Leitungsanästhesie bezeichnet man die gezielte Blockade eines Nervenstammes, der ein definiertes Gebiet versorgt (Kochs 2008). Für alle peripheren Nervenblockaden gelten folgende Kontraindikationen (Deutsche Interdisziplinäre Vereinigung für Schmerztherapie 2009, Graf und Martin 2001):

Tabelle 1

\begin{tabular}{ll}
\hline Kontraindikationen zur Anlage peripherer Nervenblockaden \\
\hline absolut & Ablehnung durch den Patienten \\
& Infektion an der Punktionsstelle \\
& Bekannte Allergie gegen Lokalanästhetika \\
& Gerinnungsstörungen \\
relativ & Vorbestehende Nervenschädigung (genaue Dokumentation \\
& erforderlich) \\
\hline
\end{tabular}

Um die Nervenleitung im peripheren Nerven zu unterbrechen und eine Anästhesie zu erreichen, werden Lokalanästhetika verwendet. Die genaue Wirkungsweise der Lokalanästhetika wurden erstmals von Ritchie et al. im Jahr 1965 als reversible Blockade des schnellen Natrium-Einstroms während der Erregungsleitung identifiziert (Ritchie et al. 1965). Nach ihrer chemischen Struktur lassen sich Lokalanästhetika in Aminoamide (Amid-Typ) und Aminoester (Ester-Typ) einteilen. Ami- 
noester-Lokalanästhetika sind Kokain, Procain, Benzocain, Tetracain und Chlorprocain. Zu den Aminoamid-Lokalanästhetika gehören Lidocain, Ropivacain, Bupivacain, Prilocain und Mepivacain. Mit Ausnahme des Benzocains sind alle Lokalanästhetika schwache Basen.

Um ihre Wirkung am Natrium-Kanal zu entfalten, müssen die Lokalanästhetika zunächst in ungeladener Form durch die Zellmembran diffundieren. Im Inneren der Nervenzelle kommt es dann zu einer Dissoziation in die geladene kationische Wirkform des Lokalanästhetikums, das an die $\alpha 1$-Untereinheit des Natriumkanals bindet und diesen blockiert. Dadurch kommt es zu einer Abnahme des Aktionspotentials, einer Abnahme der Anstiegsgeschwindigkeit des Aktionspotentials, einer Erhöhung der Depolarisationsschwelle, einer Abnahme der Leitungsgeschwindigkeit und einer Verlängerung der Refraktärzeit. Wirkstärke, Wirkdauer und die Anschlagzeit der verschiedenen Lokalanästhetika werden durch die Lipidlöslichkeit, die Proteinbindung und den Ionisationsgrad bestimmt. Hohe Lipidlöslichkeit und eine hohe Proteinbindung führen zu einer verlangsamten Anschlagzeit, zu einer verlängerten Wirkdauer und zu einem höheren Wirkungsgrad (Potenz). Niedrige Lipidlöslichkeit und Proteinbindung führen dementsprechend zu einem schnellen Wirkungseintritt mit kurzer Wirkdauer mit einer geringeren Potenz. Die Bindung an den Natriumkanal ist reversibel und der Abbau erfolgt beim Ester-Typ durch die Plasmacholinesterase. Lokalanästhetika vom Amid-Typ werden in erster Linie über das Cytochrom p450-System in der Leber metabolisiert (Dullenkopf und Borgeat 2003).

Lokalanästhetika haben verschiedene Nebenwirkungen. Allergische Reaktionen sind selten und entstehen häufiger bei Aminoester-Lokalanästhetika. Benzocain und Prilocain haben dosisabhängig das Potential zur Bildung von Methämoglobin durch Oxydation von Hämoglobin-Eisen (Dullenkopf und Borgeat 2003). In höheren Dosierungen wurde eine direkte Neurotoxizität und Myotoxizität von Lokalanästhetika beschrieben (Heavner 2007). Davon abzugrenzen sind die Nervenläsionen durch eine intraneurale Injektion. Als wichtigste Nebenwirkung bzw. Kompli- 
kation ist jedoch die systemische Wirkung von Lokalanästhetika zu nennen, da Lokalanästhetika nicht nur an Natrium-Kanäle in peripheren Nerven binden können, sondern auch in Herz-, Muskel- und Nervenzellen im Gehirn wirksam werden können. Die Auswirkungen sind von der Plasmakonzentration der Lokalanästhetika abhängig und sind am dramatischsten, wenn es zu einer akzidentellen intravasalen Injektion kommt. Die neurologischen Symptome einer systemischen Lokalanästhetika-Wirkung sind periorales Kribbeln oder Taubheitsgefühle, Kopfschmerzen und Benommenheit, Schwindel und Ohrensausen, Sehstörungen, Sprechstörungen, Muskelzuckungen, Verwirrtheit bis zur Bewusstlosigkeit, Krampfanfälle und Koma mit Atemstillstand. Die kardialen Symptome treten nach den ersten neurologischen Symptomen auf und umfassen Hyper- und Hypotension, EKG-Veränderungen, Tachykardie/Bradykardie, AV-Dissoziationen, Myokardischämien bis zur Asystolie und Kreislaufstillstand (Dullenkopf und Borgeat 2003, Zink und Graf 2003).

\subsection{Prinzip der Nervenstimulation}

Entscheidend für den Erfolg einer peripheren Leitungsanästhesie ist die möglichst nervennahe Applikation des Lokalanästhetikums. Um das zu erreichen, wird zum Beispiel ein Nervenstimulator mit einer immobilen Stimulationskanüle verwendet. Das Prinzip der Nervenstimulation wurde erstmals vom Tübinger Chirurgen Georg Perthes 1912 beschrieben, erste praktische Erfahrungen wurden jedoch erst in den 60er und 70er Jahren gemacht (Greenblatt und Denson 1962; Chapman 1972). Bei der Nervenstimulation werden niedrig frequente, kurze Impulse mit niedrigen Reizstärken auf den Nerven übertragen, die zu einer Depolarisation motorischer Efferenzen oder sensibler Afferenzen führen. Das wird erreicht, indem ein elektrischer Strom zwischen einer differenten Elektrode (Stimulationskanüle) und einer indifferenten Hautelektrode erzeugt wird. Dabei ist nach dem Coulomb-Gesetz: $\mathrm{I}=\mathrm{KxQ} / \mathrm{r} 2$ (I=Stromstärke, $\mathrm{K}=$ Konstante, $\mathrm{Q}=$ minimale Stimulationsstärke, $\mathrm{r}=$ Distanz zwischen Elektrode und Nerv) die benötigte Stromstärke umgekehrt propor- 
tional zum Quadrat des Abstandes zwischen Elektrode und Nerv. Anders formuliert heißt das, dass man für eine gleich starke Reizantwort weniger Strom benötigt, je näher die Nadelspitze am Nerven ist (Schulz-Stübner 2003). Damit ist es möglich, bei ausreichender Kenntnis der Anatomie, die Nähe der Nadelspitze an dem gewünschtem Nerv zu erkennen (Birnbaum et al. 2007). Durch Einstellung der Impulsbreite auf 0,1 ms ist es möglich, selektiv motorische Fasern zu stimulieren und damit die gewünschte motorische Reizantwort zu erhalten, ohne dem Patienten durch Stimulation sensibler Fasern Schmerzen zu bereiten. Die Frequenz wird in der Regel zwischen 1-2 Hz eingestellt (De Andrés und Sala-Blanch 2001, Neuburger et al. 2001).

\subsection{Interskalenäre Plexusanästhesie}

Bei der interskalenären Plexusanästhesie wird der Plexus brachialis in seinem Verlauf zwischen dem Musculus scalenus anterior und dem Musculus scalenus medius - der sogenannten Skalenuslücke - anästhesiert und stellt damit den kranialsten Zugang zum Plexus brachialis dar. Der Plexus brachialis bildet sich aus den Rami anteriores der Spinalnerven C5-Th1. Zwischen dem Musculus scalenus anterior und dem Musculus scalenus medius laufen sie zusammen und bilden drei Trunci aus: Den Truncus superior (aus C5 und C6), den Truncus medius (aus C7) und den Truncus inferior (aus C8 und Th1). Aus den Ästen der Trunci entstehen der Fasciculus posterior, der Fasciculus lateralis und der Fasciculus medialis. Die 3 Faszikel folgen dem Verlauf der Arteria subclavia und erreichen zwischen der Klavikula und der ersten Rippe die Achselhöhle. Der Plexus brachialis ist mit den BlutgefäBen von kollagenem Bindegewebe umgeben (Schulz-Stübner 2003, Hempel 1999). Die interskalenäre Plexusanästhesie wurde erstmals von A. P. Winnie 1970 beschrieben und wurde von G. Meier 1997 durch Veränderung der Punktionsstelle und des Einstichwinkels modifiziert, um Komplikationen wie eine intraspinale Injektion zu vermeiden und die Einlage eines Katheters zu ermöglichen (Winnie 1970, Meier et. al. 1997). Mit der interskalenären Plexusanästhesie erreicht man 


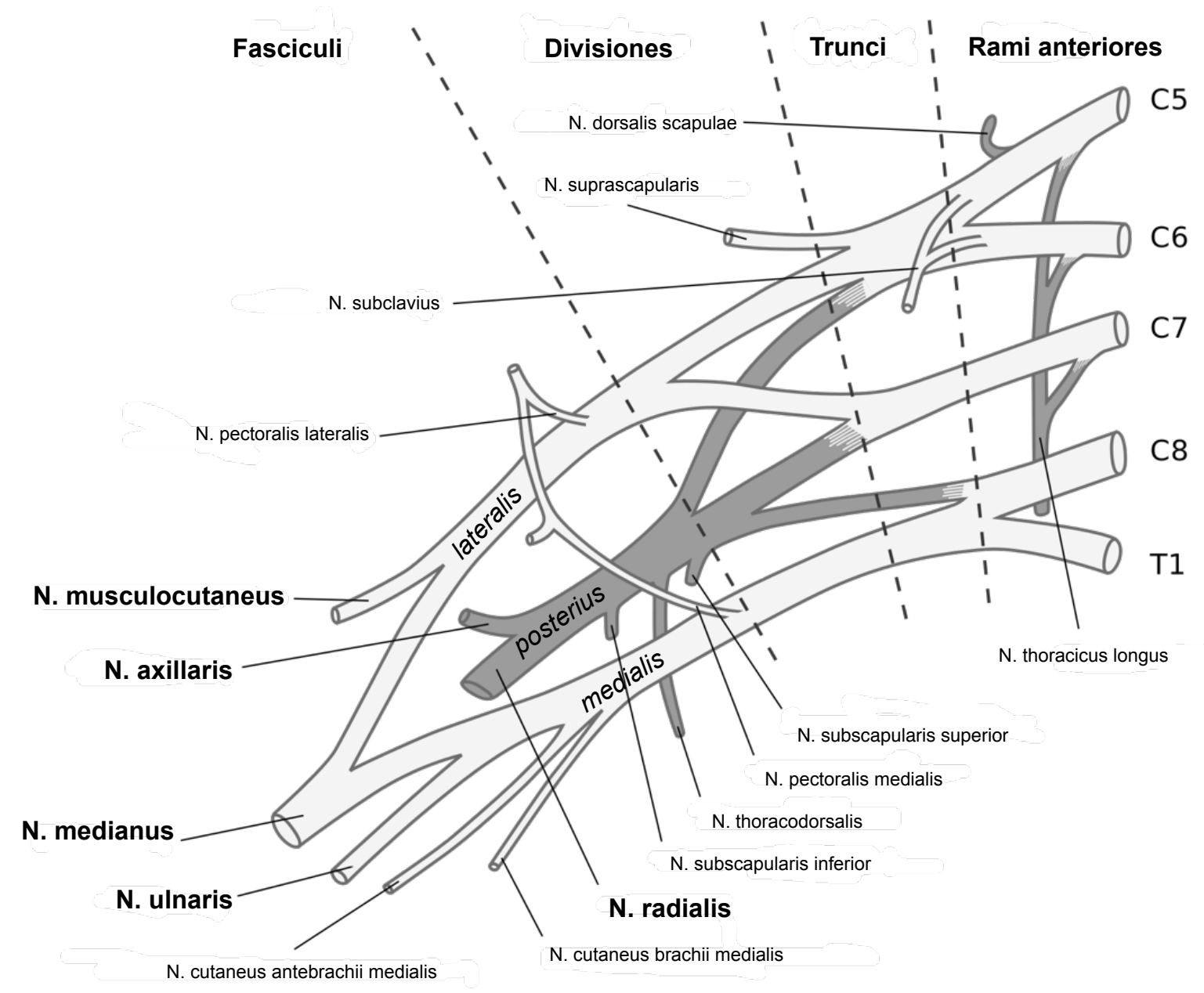

Abbildung 1: Plexus brachialis (modifiziert nach H. Gray 1918 „Anatomy of the Human Body“, Original: www.bartleby.com/107/210.html)

eine Anästhesie im Oberarm und Schulterbereich. Die Innenseite des Armes wird durch meist unzureichende Betäubung des Nervus ulnaris nicht sicher erreicht. Indikationen für dieses Verfahren sind Schmerztherapie und chirurgische Eingriffe an Schulter und proximalen Oberarm wie Schulterarthroskopie und Reposition von Schultergelenksluxationen (Hempel 1999, Meier et al. 2001). Neben den o.g. allgemeinen Kontraindikationen bei peripheren Nervenblockaden, gibt es spezielle Kontraindikationen für die interskalenäre Plexusanästhesie wie das Vorliegen einer kontralateralen Phrenicus- oder Rekkurensparese, bzw. eine relative Kontraindikation bei Patienten mit einer COPD, die sich aus den folgenden Komplikationen ergeben (Meier et al. 2001). Neben seltenen Komplikationen wie eine arterielle Fehlpunktion, intravasale Lokalanästhetika-Injektion oder iatrogener Pneumothorax (Borgeat et al. 2001, Neuburger und Büttner 2011), können bei der interska- 
lenären Plexusanästhesie - durch die anatomische Nähe des Nervus Phrenikus, des Nervus laryngeus recurrens und des Ganglion stellatum - diese durch Diffusion des Lokalanästhetikums betäubt werden und folgende Probleme verursachen.

Durch die Blockierung des N. Phrenikus kommt es zu einer ipsilateralen Parese des Zwerchfells. In mehreren Studien konnte bis zu 100\% eine Phrenikusparese mit Einschränkung der Vitalkapazität nachgewiesen werden, ohne aber immer eine klinisch relevante Störung der Ventilation zur Folge zu haben (Urmey et al. 1991, Fujimura et al. 1995, Sala-Blanch et al. 1999, Hortense et al. 2010, Neuburger und Büttner 2011). Die Inzidenz einer klinisch relevanten Parese ist in der Literatur unterschiedlich beschrieben worden und bewegt sich zwischen 3,3\% und 10\% (Meier et al. 1997, Schwemmer et al. 2004, Brandl und Taeger 1991).

Durch die Betäubung des Ganglion stellatum kann es zu einem Horner-Syndrom (Ptosis, Myosis, Enophtalmus) kommen und dies ist in 5\% bis 75\% der Fälle beschrieben (Brown et al. 1993, Meier et al. 1997, Brandl und Taeger 1991, VesterAndersen et al. 1981, Neuburger und Büttner 2011).

Eine Parese des Nervus laryngeus reccurens mit Heiserkeit kommt in 1\% bis 6,5\% der Fälle vor (Brown et al. 1993, Meier et al. 1997, Brandl und Taeger 1991). Respiratorische Probleme durch eine beidseitige Parese sind selten und nur in Einzelfällen beschrieben (Seltzer 1977, Kempen et al. 2000). Ein vorübergehendes neurologisches Defizit wird in 2,3\% - 2,84\% der Fälle beschrieben (Bishop et al. 2005, Brull et al. 2007). Nervenschäden nach Operationen mit peripheren Regionalanästhesie-Verfahren haben unterschiedliche Ursachen und können in $88 \%$ der Fälle auf die Operation zurückgeführt werden (Horlocker et al. 1999). Ursachen von Nervenläsionen bei peripheren Regionalanästhesie-Verfahren können direkte toxische Effekte der Lokalanästhetika, Nervenkompression durch schnelle Applikation, Ödembildung und intrafaszikuläre Injektionen sowie Verletzungen des Perineuriums und Ischämien sein (Hogan 2008). Die Inzidenz der Nervenschäden ist unklar, da es keine einheitlichen Definitionen des Nervenschadens gibt und wegen der guten Prognose und der Rückläufigkeit der Symptome lange Beobachtungszeiträume 
nötig sind (Neuburger und Büttner 2011). Die Inzidenz von Nervenläsionen nach Regionalanästhesieverfahren wird je nach Studie mit $0,02 \%$ bis $0,04 \%$ angegeben, wobei permanente Nervenschäden nur in Einzelfällen beschrieben wurden (Barrington et al. 2009, Auroy et al. 2002, Auroy et al. 1997, Capdevila et al. 2005, Brull et al. 2007). In Bezug auf die interskalenäre Plexusanästhesie ist lediglich ein Fall eines permanenten Nervenschadens von Bishop et al. beschrieben worden (Bishop et al. 2005).

Die sicherlich schlimmste Komplikation, die bei der Anlage einer interskalenären Plexusanästhesie auftreten kann, ist die intraspinale oder intraneurale Fehlpunktion, die einen permanenten Funktionsverlust der Nerven des gesamten Plexus brachialis auf spinaler Ebene zur Folge haben kann. Dazu sind vereinzelte Fallberichte veröffentlicht. Vermehrt traten diese Fälle auf, nachdem die interskalenäre Plexusanästhesie in Allgemeinanästhesie durchgeführt worden war. Daher soll die Anlage einer interskalenären Plexusanästhesie nur bei wachen Patienten durchgeführt werden (Kumar et al. 1971, Ross und Scarborough 1973, Passannante 1996, Benumof 2000, Walton et al. 2000).

\subsection{Narkoseverfahren für arthroskopische Schulteroperationen}

In den letzten Jahren sind zahlreiche Studien veröffentlicht worden, die die interskalenäre Plexusanästhesie bei arthroskopischen Schulteroperationen untersuchten. Grundsätzlich verspricht die Durchführung einer peripheren Leitungsanästhesie Vorteile gegenüber der Allgemeinanästhesie wie weniger postoperative Schmerzen, geringere Inzidenz von postoperativer Übelkeit und Erbrechen, höhere Patientenzufriedenheit und schnellere Entlassung aus dem Aufwachraum (Woolf und Chong 1993, Chan et al. 2001, McCartney et al. 2004, Klein et al. 2002, Hadzic et al. 2005, O'Donnell et al. 2008).

Diese Erkenntnisse sind auch für die interskalenäre Plexusanästhesie bei Operationen an der Schulter beschrieben worden (Brown et al. 1993, Arciero et al. 1996, D'Alessio et al. 1995a, Stiglitz et al. 2011). Es scheint auch ökonomische Vorteile 
gegenüber der Allgemeinanästhesie zu geben, dies wird aber kontrovers diskutiert (Greenberg 1995, Schuster et al. 2005, Horn et al. 2007, Gonano et al. 2009, Williams et al. 2005), zumal der Zeitaufwand zur Anlage der interskalenären Plexusanästhesie von der Erfahrung des Anästhesisten abhängig ist (Arciero et al. 1996).

Die Akzeptanz der Patienten von Regionalanästhesie-Verfahren wird unterschiedlich beschrieben und ist von verschiedenen Faktoren abhängig. 1991 beschrieben Shevade und Panagopoulos, dass 66\% der Patienten eine Allgemeinanästhesie bevorzugen, da sie während der Operation ,nichts sehen oder fühlen“ wollen (Shevade und Panagopoulos 1991). Mattey et al. beschrieben, das 49,3\% der Patienten eine Allgemeinanästhesie bevorzugen würden. Auf die Frage nach Befürchtungen in Bezug auf Regionalanästhesie-Verfahren hatten 26,7\% der Patienten große Befürchtung bzw. 29\% geringe Befürchtung etwas von der Operation zu sehen (Matthey et al. 2004). Grundsätzlich ist die Präferenz der Patienten abhängig von der Aufklärung des Anästhesisten und von bereits gemachten Erfahrungen mit Allgemein- oder Regionalanästhesie (Webster et al. 2011). Ein besonderes Problem bei der interskalenären Plexusanästhesie allein - und damit bei einem wachen Patienten - bei einer Schulteroperation stellt die Nähe des Operationsgebietes zum Kopf des Patienten dar. Dadurch ist der Zugang des Anästhesisten zum Kopf eingeschränkt und die Akzeptanz des Patienten vermeintlich geringer (Grossi et al. 1998). Daher wird von einigen Autoren eine Kombination aus interskalenärer Plexusanästhesie mit einer Allgemeinanästhesie bevorzugt. Bei der Kombination aus beiden Verfahren kann der Vorteil der interskalenären Plexusanästhesie in Bezug auf postoperative Schmerzen genutzt werden und zudem werden intraoperativ weniger Analgetika benötigt (Brandl und Taeger 1991, Grossi et al. 1998). Da Opioide vor allem in höherer Dosierung einen emetogenen Effekt haben, kann dies bei prädisponierten Patienten einen positiven Einfluss auf das postoperative Erbrechen haben (Apfel und Roewer 2000).

Oft werden schulterchirurgische Eingriffe in halb-sitzender beach-chair-Position durchgeführt. Diese Position geht unabhängig vom Narkoseverfahren mit einer ho- 
hen Inzidenz von Hypotension und damit mit einen Risiko einer insuffizienten zerebralen Perfusion einher (Lee et al. 2011, Yadeau et al. 2011, Jeong et al. 2012). Dieser Blutdruckabfall scheint unter Verwendung der interskalenären Plexusanästhesie geringer, es ist aber das Auftreten des Bezold-Jarisch-Reflexes in 13-28\% der Fälle beschrieben worden, nachdem die Patienten in die beach-chair-Position gebracht worden waren (D'Alessio et al. 1995b, Liguori et al. 1998, Kahn und Hargett 1999, Trentman et al. 2011). Dieser kardioinhibitorische Reflex ist gekennzeichnet durch schwere Bradykardie mit Hypotension und Vasodilatation, was bis zum Herzstillstand gehen kann und geht auf Beschreibungen von Bezold und Hirt aus dem Jahr 1867 sowie Jarisch und Richter aus dem Jahr 1939 zurück, die bei der intravenösen Applikation von Veratrum-Alkaloiden bei Katzen die o.g. hämodynamischen Effekte in Kombination mit einer Apnoe beobachteten. Vermittelt wird der Reflex über chemo- und mechanosensitive Rezeptoren in der Innenwand der Ventrikel des Herzens. Als auslösende Faktoren gelten neben chemischen Stoffen wie Alkaloiden, Adenosintriphosphat, Capsaicin und Schlangen-Giften vor allem ein verminderter venöser Zustrom zum Herzen, zu dem es bei einer sitzende Lagerung kommt sowie psychologische Auslöser wie Angst und Schmerzen (Campagna und Carter 2003, Kinsella und Tuckey 2001). 


\subsection{Fragestellung}

Die vorliegende Studie vergleicht die interskalenäre Plexusanästhesie in Kombination mit einer total-intravenösen Anästhesie mit der total-intravenösen Anästhesie allein für ambulante Schulteroperationen, um folgende Fragestellungen zu beantworten:

- Ergeben sich Vor- oder Nachteile durch die Kombination aus interskalenärer Plexusanästhesie und total-intravenöser Anästhesie in Bezug auf postoperative Komplikationen wie postoperative Übelkeit und Erbrechen (PONV), Shivering und Schmerzen?

- Kann durch die Kombination von Allgemeinanästhesie und interskalenärer Plexusanästhesie der Schmerzmittelbedarf der Patienten nach Entlassung nach Hause gesenkt werden?

- Wie verhalten sich die Prozesszeiten bei der Verwendung einer interskalenären Plexusanästhesie im Vergleich zu Allgemeinanästhesie allein?

- Wie ist die Akzeptanz und die Zufriedenheit der Patienten mit der Kombination aus Allgemeinanästhesie mit einem Regionalanästhesie-Verfahren?

- Kann durch die Kombination von Allgemeinanästhesie und interskalenärer Plexusanästhesie der intraoperative Schmerzmittelbedarf gesenkt werden?

- Beeinflusst das Narkoseverfahren den Erfolg der Operation? Wie schätzen die Patienten den Erfolg der Operation ein?

- Ergeben sich Vor- oder Nachteile durch die Kombination aus interskalenärer Plexusanästhesie und total-intravenöser Anästhesie in Bezug auf die intraoperative Hämodynamik der Patienten? Kommt es zu einem Auftreten des Bezold-Jarisch-Reflexes? 


\section{Material und Methoden}

Zur vorliegende Studie liegt ein Votum der Ethik-Kommission der Universität Göttingen vor und sie ist im deutschen Register klinischer Studien registriert (DRKS00005295). Alle Patienten, die sich in einer ambulanten orthopädischen Praxis im Zeitraum 2008 bis 2010 einer Arthroskopie des Schultergelenkes unterzogen haben, wurden in dieser Untersuchung berücksichtigt. Indikationen für die Operationen waren Impingement-Syndrom, Verletzungen der Rotatorenmanschette und der Bizepssehne sowie die diagnostische Arthroskopie. Insgesamt wurden 83 Patienten in die Untersuchung einbezogen.

Bei 41 Patienten wurde der Eingriff in Allgemeinanästhesie (VN), bei 42 Patienten wurde zusätzlich zu der Allgemeinanästhesie eine interskalenäre Plexusblockade (VN+ISB) durchgeführt. Die Randomisierung und die Durchführung der interskalenäre Plexusanästhesie erfolgte durch den zuständigen Facharzt der ambulanten Anästhesieeinrichtung. Patienten mit Kontraindikationen zur Anlage der interskalenären Plexusanästhesie oder bei erfolgloser Blockade wurden dann in der VNGruppe eingeschlossen. Die Einwilligung zur anonymisierten Auswertung der perioperativen Daten gaben die Patienten mit der Einwilligung in die Narkose. Die Durchführung und Aufrechterhaltung der Allgemeinanästhesie wurde von verschiedenen Anästhesisten unter Aufsicht des zuständigen Facharztes durchgeführt. Die nachträgliche Auswertung der perioperativen Daten erfolgte durch einen verblindeten Untersucher.

Die ambulante Anästhesieeinrichtung führte ab dem zweiten postoperativen Tag eine Patientenbefragung durch, um die Zufriedenheit der Patienten zu quantifizieren. Dabei konnten 73 Patienten erfolgreich befragt werden, 10 Patienten haben die Befragung abgelehnt. Die Entwicklung des Fragebogens erfolgte in Zusammenarbeit mit dem Zentrum für Anästhesie, Rettungs- und Intensivmedizin der Universitätsmedizin Göttingen. Die Auswertung der Befragung erfolgte anschließend durch einen verblindeten Untersucher. 


\subsection{Interskalenäre Plexusanästhesie}

Zur Durchführung der interskalenären Plexusanästhesie wurde der Nerven-Stimulator Stimuplex ${ }^{\circledR}$ HNS 11 der Firma Braun (Melsungen, Deutschland) verwendet. Das Gerät bietet die Möglichkeit zur percutanen und transcutanen Stimulation. Es können verschiedene Impulsbreiten $(0,1 \mathrm{~ms}, 0,3 \mathrm{~ms}, 0,5 \mathrm{~ms}$ und $1,0 \mathrm{~ms})$ ausgewählt werden und damit selektiv motorische und sensorische Nervenfasern gereizt werden. Die Stromstärke ist in 0,1 mA-Schritten von 0 bis maximal $60 \mathrm{~mA}$ einstellbar. Die Frequenz der Impulse ist zwischen 1 und $2 \mathrm{~Hz}$ wählbar. Das Display zeigt die eingestellte Impulsbreite, den eingestellten Impulsstrom sowie den tatsächlich durch den Patienten fließenden Strom an. Zur Punktion wurde die atraumatische single-shot Stimulationskanüle (Stimuplex $\mathrm{D}^{\circledR}, 22 \mathrm{G} 30^{\circ}$ ) der Firma Braun verwendet.

Die Durchführung der interskalenären Plexusanästhesie in der untersuchten ambulanten Anästhesieeinrichtung folgte einem Standardvorgehen. Dabei wurde der Patient auf dem Rücken gelagert, und der Kopf wurde leicht auf die Gegenseite gedreht. Der Patient erhielt über eine Gesichtsmaske 4 Liter Sauerstoff pro Minute

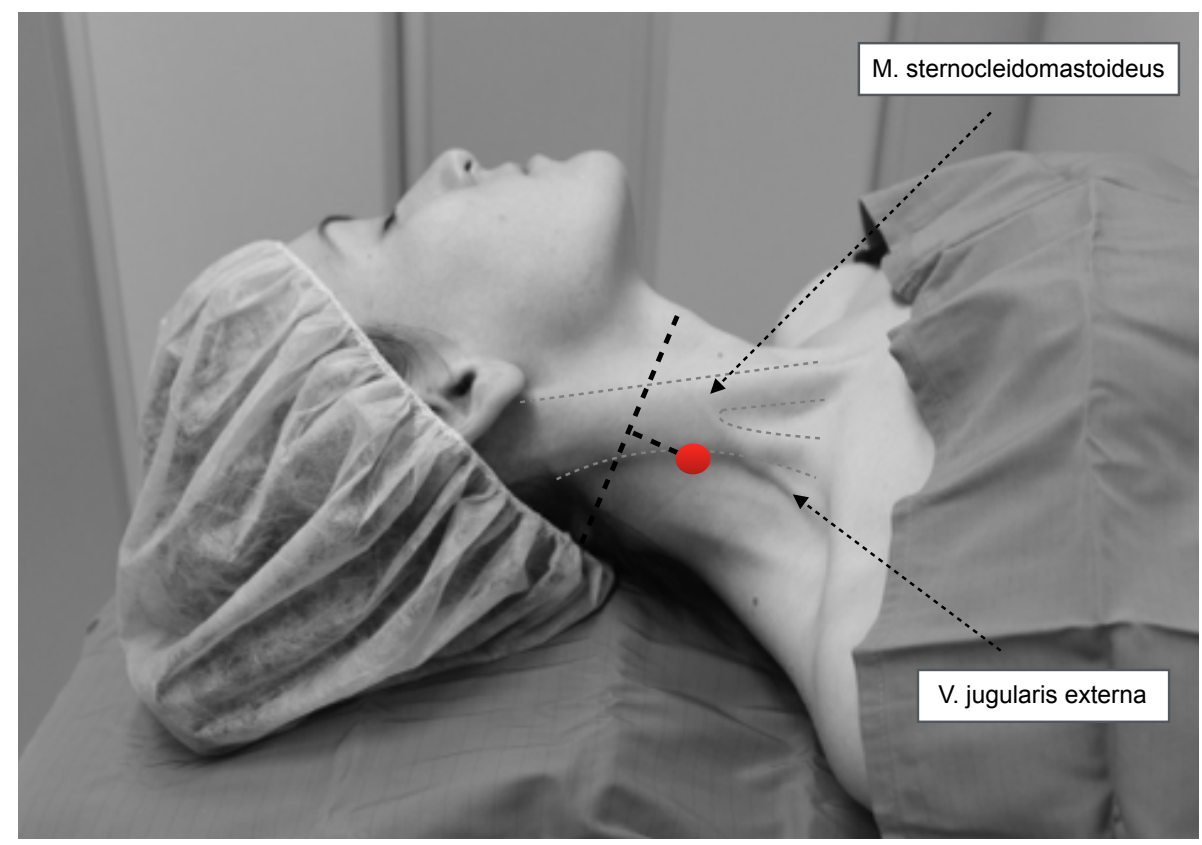

Abbildung 2: Punktionsstelle zur interskalenären Plexusanästhesie; Landmarken nach Meier (Foto: H. Janssen) 
und der Kreislauf wurde mithilfe von EKG, Pulsoxymetrie und nicht-invasiver Blutdruckmessung (NIBP) überwacht. Der Patient erhielt einen peripheren Venenzugang und es wurde eine Analgosedierung mit Remifentanil (Ultiva ${ }^{\circledR}$, GlaxoSmithKline AG, Hamburg, Deutschland) mit $0,05 \mu \mathrm{g} / \mathrm{kgKG} / \mathrm{min}$ und Midazolam (Dormicum $^{\circledR}$, Fa. Hoffmann-La Roche AG, Basel, Schweiz) mit 0,05 mg/kgKG durchgeführt. An der Einstichstelle erfolgte eine Lokalanästhesie mit $2 \mathrm{ml}$ Mepivacain $1 \%$ s.c.(Meaverin ${ }^{\circledR}$, Fa. DeltaSelect GmbH, Pfullingen, Deutschland).

Die Einstichstelle befindet sich am lateralen Rand des Musculus sternocleidomastoideus, ca. $2 \mathrm{~cm}$ unterhalb der Höhe der Incisura thyroidea auf der Höhe des 6 . Halswirbels (Landmarken nach Meier). Diese Angaben gelten für einen „Normalpatienten“ (175 cm; $75 \mathrm{~kg})$ und müssen den individuellen Gegebenheiten das Patienten angepasst werden. Zur besseren Tastbarkeit des Musculus sternocleidomastoideus kann man den Patienten bitten den Kopf zu heben, wodurch sich der Muskel kontrahiert und damit besser zum Vorschein kommt. Die Stichrichtung liegt im Verlauf der Skalenuslücke, leicht nach latero-caudal in die Richtung der Grenze zwischen lateralem und medialem Klavikuladrittel, mit einem Einstichwinkel von ca. $30^{\circ}$ zur Haut. Nach einminütiger Desinfektion des Hautbereichs mit einem alkoholischen Desinfektionsmittel (Kodan ${ }^{\circledR}$, Schülke\&Mayr, Norderstedt, Deutschland) erfolgte die Punktion mit einer $50 \mathrm{~mm} 22 \mathrm{G}$ Stimuplex $\mathrm{D}^{\circledR}$ der Firma Braun. Der angeschlossene Nervenstimulator (Stimuplex ${ }^{\circledR}$ HNS 11, Fa. Braun, Melsungen, Deutschland) ist mit einer Stromstärke von $2 \mathrm{~mA}$, einer Frequenz von $1 \mathrm{~Hz}$ und einer Impulsbreite von $0,1 \mathrm{~ms}$ eingestellt. Dadurch ist es möglich selektiv motorische A $\alpha$-Fasern zu stimulieren. Sensorische Fasern werden nicht gereizt, was eine schmerzlose Nervenstimulation möglich macht. Die Gefäß-Nerven-Scheide wird nach etwa $2 \mathrm{~cm}$ bis maximal $5 \mathrm{~cm}$ erreicht. Die gewünschte Reizantwort des Musculus deltoideus und des Musculus bizeps brachii wird bei Erreichen des Truncus superior des Plexus brachialis erzielt. Die korrekte Position der Punktionsnadel ist erreicht, wenn bei unter 0,3 mA eine deutliche Reizantwort zu sehen ist. Gibt es eine Reizantwort unterhalb von $0,2 \mathrm{~mA}$ ist die Nadel zu nah am Nerven und die 
Nadel wird vorsichtig zurückgezogen, um eine intraneurale Injektion zu vermeiden (Schulz-Stübner 2003, Neuburger et al. 2001, Meier et al. 2001). War die Nadel korrekt platziert, wurden nach einem negativen Aspirationstest $40 \mathrm{ml}$ Mepivacain $1 \%$ (Meaverin ${ }^{\circledR}$, Fa. Deltaselect, Pfullingen, Deutschland) injiziert. Der Erfolg der Anästhesie wurde anschließend mit Testung der Temperaturempfindung mithilfe von Eis kontrolliert. Dokumentiert wurden neben den verwendeten Materialien und dem Punktionsort auch die niedrigste benötigte Stromstärke zur Stimulation, Tiefe der Nadelspitze unter Hautniveau, Auslösung oder Nichtauslösung von Parästhesien sowie Besonderheiten bei der Punktion. Anschließend erfolgte die Einleitung der Narkose.

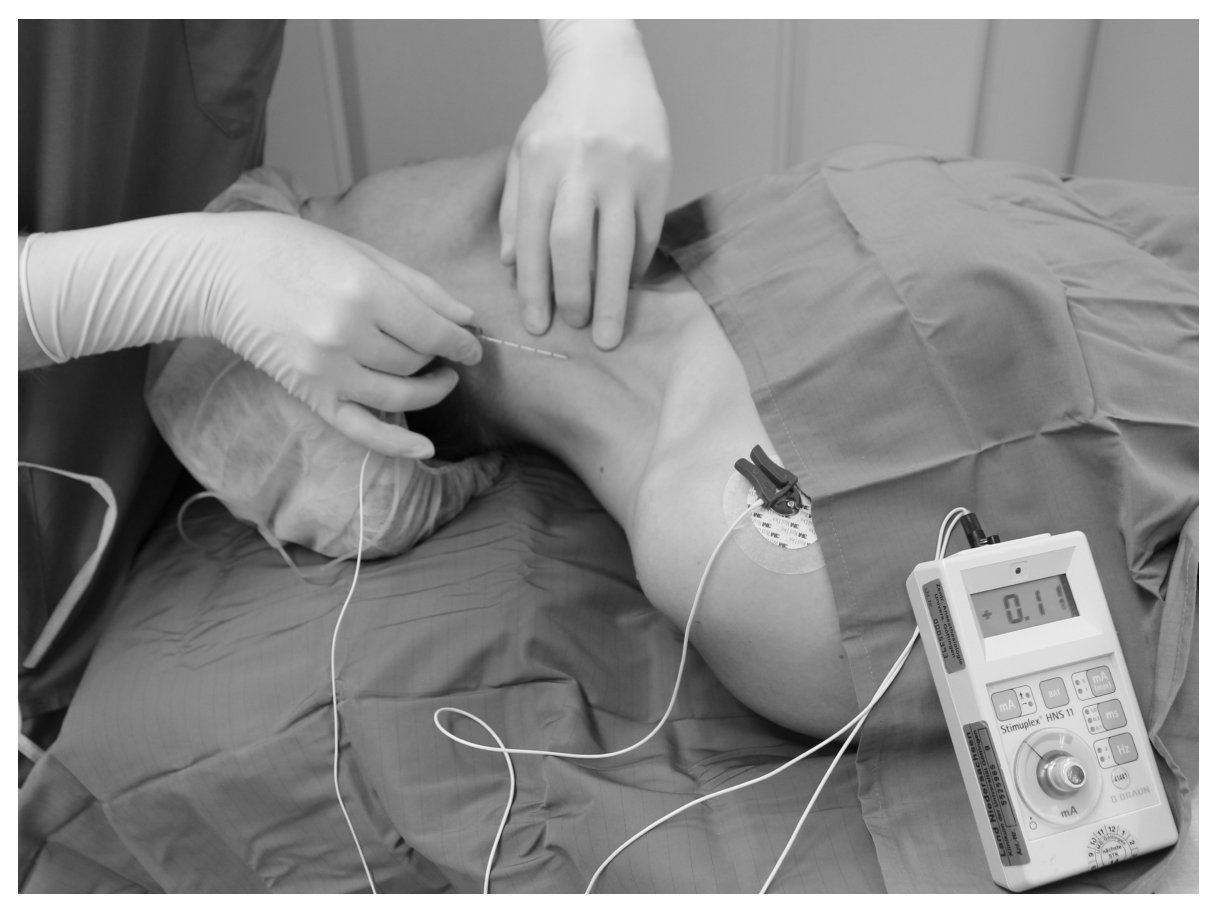

Abbildung 3: Demonstration der Durchführung einer interskalenären Plexusanästhesie mit angeschlossenem Nervenstimulator (Foto: $\mathrm{H}$. Janssen)

\subsection{Allgemeinanästhesie}

Die Durchführung der total intravenösen Anästhesie erfolgte in beiden Patientengruppen nach dem gleichen Standardvorgehen. Dazu wurde der Patient gelagert und das Standardmonitoring (EKG, Pulsoxymetrie und nicht-invasive Blutdruckmessung) angeschlossen. Wenn noch nicht erfolgt, wurde ein peripherer Venenzugang gelegt und eine Infusion $(\mathrm{NaCl}$ 0,9\%, $500 \mathrm{ml})$ angeschlossen. Gegebenenfalls 
wurde vor Einleitung der Narkose Midazolam (Dormicum ${ }^{\circledR}$, Fa. Hoffmann-La Roche AG, Basel, Schweiz) zur Prämedikation gegeben. Anschließend erfolgte die 3minütige Präoxygenierung, bevor die Narkose eingeleitet wurde.

Zur Analgesie wurde Remifentanil (Ultiva ${ }^{\circledR}$, GlaxoSmithKline, Hamburg, Deutschland) mit $1 \mu \mathrm{g} / \mathrm{kgKG} / \mathrm{min}$ über 30 Sekunden oder Sufentanil (Sufenta mite $10^{\circledR}$, Janssen-Cilag GmbH, Neuss, Deutschland) mit 0,2 bis $0,3 \mu \mathrm{g} / \mathrm{kgKG}$ verwendet. Bei einigen Narkosen wurde auch Alfentanil (Rapifen ${ }^{\circledR}$, Janssen-Cilag GmbH, Neuss, Deutschland) mit einer Dosierung von 15-20 $\mu \mathrm{g} / \mathrm{kgKG}$ verwendet. Häufig wurden auch mehrere Opioide kombiniert eingesetzt. Außerdem wurde teilweise SKetamin als Nicht-Opioid-Analgetikum verwendet.

Die Einleitung der Narkose erfolgte dann mit Propofol (Propofol 1\%, Fresenius Kabi Deutschland GmbH, Bad Homburg, Deutschland) mit $2 \mathrm{mg} / \mathrm{kgKG}$ über 2 Minuten, unter Kontrolle des BIS-Monitors. Wurde ein BIS-Wert unter 60 erreicht, wurde die Induktion gestoppt. Nach erfolgreicher Einleitung und sichergestellter manueller Beatmung über Maske wurde der Patient mit Mivacurium (Mivacron $^{\circledR}$, Fa. GlaxoSmithKline, Hamburg, Deutschland) 0,2 $\mathrm{mg} / \mathrm{kgKG}$ relaxiert. Dann erfolgte die orotracheale Intubation mittels direkter Laryngoskopie mit einem Spiral-Tubus (Ruesch Flex-Spiraltubus ${ }^{\circledR}$, Fa. Teleflex Medical GmbH, Kernen, Deutschland) und der Tubus wurde mithilfe eines Cuffdruck-Messers geblockt. Bei Männern wurde in der Regel ein Tubus mit einem Innendurchmesser von 8-8,5 mm, bei Frauen ein Innendurchmesser von 7-7,5 mm gewählt. Nach Sicherstellung der korrekten Tubuslage mittels Kapnometrie und Auskultation wurde ein Oropharyngealtubus (Guedel-Tubus) eingelegt, der Spiral-Tubus wurde fixiert und das Beatmungsgerät wurde angeschlossen. Bevor die Patienten in die beach-chairPosition gebracht wurden, wurden ggf. $500 \mathrm{ml}$ kolloidale Infusion (Hydroxyethylstärke, HAES 6\%) verabreicht, um eine lagerungsbedingte Hypotonie zu vermeiden. Dann wurde der Patient gelagert und war zur Operation freigegeben. Die Narkosetiefe wurde mithilfe eines BIS (Bispectral-Index)-Monitors überwacht und zur Aufrechterhaltung der Narkose wurde Propofol mit $6-10 \mathrm{mg} / \mathrm{kgKG} / \mathrm{h}$, je nach 
BIS-Index, verwendet.

Zur Überwachung der Narkosetiefe wurde der BIS VISTA ${ }^{\circledR}$ Monitor der Firma Covidien Deutschland GmbH (Neustadt an der Donau, Deutschland) verwendet. Dazu wurde ein Stirnsensor (BIS-Quatro-Sensor ${ }^{\mathbb{R}}$ ) aufgebracht, der Roh-EEG-Daten ableitet. Das BIS-System errechnet daraus eine Zahl zwischen 0 und 100. Dabei bedeutet die Zahl „0“ das Fehlen einer elektrischen Hirnaktivität, während „100“ die vollständige Wachheit des Patienten anzeigt. Der Zielwert für eine Allgemeinanästhesie liegt zwischen 40 und 60. Auf dem Monitor kann neben dem BIS-Wert zwischen 0 und 100 auch das Roh-EEG, die Impedanz - also die Störungen durch Muskelbewegungen - und der zeitliche Verlauf der beiden Parameter abgelesen werden.

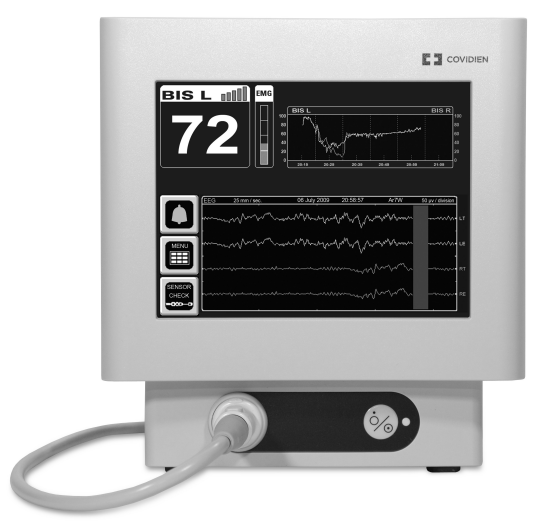

Abbildung 4: BIS VISTA ${ }^{\circledR}$ Monitor der Covidien GmbH (www.covidien.com)

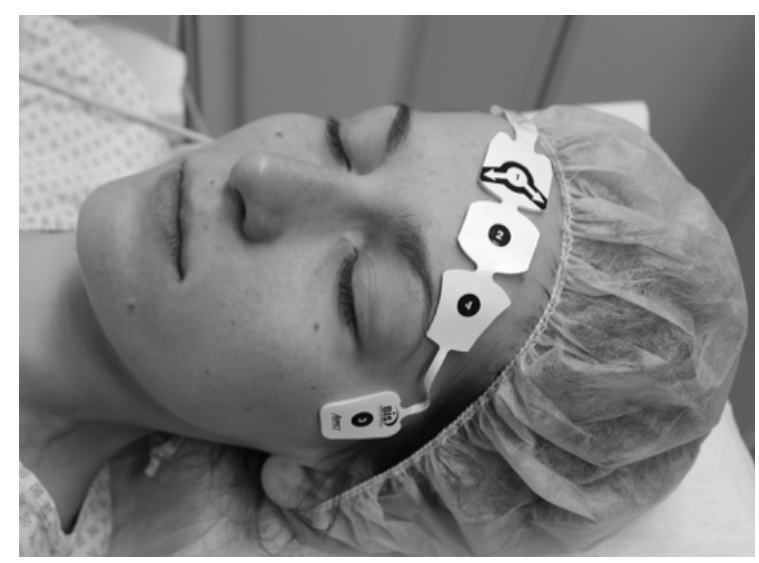

Abbildung 5: Platzierung der BISQuatro-Elekroden (Foto: H. Janssen)

\subsection{Auswertung der Narkoseprotokolle}

Ausgewertet wurden die Narkoseprotokolle von Patienten, die eine arthroskopische Operation eines Schultergelenks erhalten haben und im Zeitraum von Oktober 2008 bis Dezember 2010 in der ambulanten orthopädischen Einrichtung operiert worden sind. Erfasst wurden folgende Parameter.

Neben dem Alter der Patienten wurden die Körpergröße und das Körpergewicht erfasst. Daraus wurde der Body Mass Index (BMI) errechnet. Die Einteilung in 
Unter- und Normalgewicht bzw. Präadipositas und Adipositas Grad I-III erfolgte nach der Definition der World Health Organization (WHO) aus dem Jahre 2004. Sämtliche angegebenen Vorerkrankungen wurden ermittelt, inklusive Nikotin- und Alkoholabusus und Allergien. Außerdem wurde die Vormedikation erfasst. Nach all diesen Informationen erfolgte die Einteilung nach dem Physical Status Classification System der American Society of Anesthesiologists (ASA-Klassifikation). In Bezug auf die Narkose wurden der Narkosemittel-Verbrauch erfasst sowie die Stimulationsgrenze und die Menge des injizierten Lokalanästhetikums bei der interskalenären Plexusanästhesie. Zudem wurde die Menge der infundierten kristalloiden und kolloidalen Infusionslösungen festgehalten. Außerdem wurden etwaige Komplikationen und die damit verbundenen Medikamentengaben dokumentiert. In Bezug auf den Verlauf der Narkose wurden Vitalparameter erfasst und ausgewertet. Es wurden alle dokumentierten systolischen Blutdruckwerte erfasst. Herausgestellt wurden der Blutdruck vor Einleitung der Narkose, vor der Verbringung in die beach-chair-Position sowie der Maximalwert und der Minimalwert. Aus allen Werten wurde der Durchschnittsblutdruck mit Standardabweichung und der maximale Blutdruckabfall vom Ausgangsblutdruck errechnet und erfasst. Auch sämtliche dokumentierte Puls-Frequenzen wurden erfasst. Wie bei dem Blutdruck wurden Ausgangswert, Maximal- und Minimalwert herausgestellt. Errechnet wurden der Durchschnittswert mit Standardabweichung und die maximale Herzfrequenz-Änderung vom Ausgangswert. Die pulsoxymetrisch gemessene periphere Sauerstoffsättigung wurde erfasst und der Ausgangswert, der Maximalwert und der Minimalwert festgehalten. Errechnet wurden der Durchschnittswert mit der Standardabweichung. Außerdem wurden die perioperativen Prozesszeiten nach der gemeinsamen Empfehlung von DGAI, BDA, BDC und VOPM dokumentiert (Bauer et al. 2008). Lediglich die „Anästhesiologische Vorlaufzeit“ wurde mit dem Beginn des Patienten im Operationssaal definiert, um eine Differenzierung zwischen der Zeit zur Anlage der Regionalanästhesie und der Zeit zur Einleitung der Allgemeinanästhesie zu erreichen. Der höhere Zeitaufwand durch die Anlage der interskalenären 
Plexusanästhesie findet in der Kennzahl der „reinen Anästhesiezeit“ Berücksichtigung. Daraus resultieren folgende Definitionen der Prozesszeiten:

1. Zeit für Anlage der interskalenären Plexusanästhesie (Zeit von Beginn der Hautdesinfektion bis zum Erreichen der Blockade)

2. Anästhesiologische Vorlaufzeit (Ankunft des Patienten im Operationssaal bis Freigabe Anästhesie)

3. Schnitt-Naht-Zeit (Zeit von Beginn Hautschnitt bis zum Ende der Hautnaht)

4. Anästhesiologische Nachlaufzeit (Ende nachbereitender operativer Maßnahmen bis zum Ende der Präsenz Anästhesie-Arzt)

5. Anästhesie-kontrollierte Zeit (Anästhesiologische Vorlaufzeit plus anästhesiologische Nachlaufzeit)

6. Reine Anästhesiezeit (Zeit für Anlage der interskalenären Plexusanästhesie plus Anästhesie-kontrollierte Zeit)

\subsection{Patientenbefragung}

Im Rahmen der Qualitätssicherung führte die Praxis für Anästhesie eine Patientenbefragung von Oktober 2008 bis zum Januar 2010 durch. Diese Befragung hatte zum Ziel, die Zufriedenheit der Patienten mit der Narkose mit und ohne Kombination mit einer interskalenären Plexusanästhesie zu ermitteln und diese zu vergleichen. Zur wissenschaftlichen Auswertung der Daten liegt ein Votum der Ethikkommission der Universitätsmedizin Göttingen vor. Erfragt wurden die Schmerzen vor und nach der Operation mit dem verbundenem Schmerzmittelbedarf, das Auftreten von Nebenwirkungen wie postoperative Übelkeit (PONV) und Shivering. Die Schmerzen wurden mithilfe der numerischen Rating-Skala (NRS) ermittelt. Erfragt wurden die Schmerzen vor dem operativen Eingriff, direkt nach der Operation und am ersten und zweiten Tag nach der Operation. In Bezug auf Schmerzmittel wurde zunächst erfragt, welche Schmerzmittel die Patienten vor der Operation 
eingenommen wurden. Nach der Operation bekamen alle Patienten Schmerzmittel nach einem festen Schema verschrieben. Alle Patienten sollten 3 mal täglich Ibuprofen $600 \mathrm{mg}$ über 7 Tage und 4 mal täglich Metamizol-Tropfen mit der Dosierung von $1 \mathrm{~g}$ in den ersten beiden postoperativen Tagen einnehmen. Bei Auftreten von besonders starken Schmerzen konnten die Patienten $50 \mathrm{mg}$ Tramadol, maximal 4 mal täglich einnehmen. Es wurde erfragt, ob diese Medikamente wie verordnet eingenommen wurden, ob die Patienten auf die Bedarfsmedikation bei besonders starken Schmerzen zurückgreifen mussten, oder ob sie weniger einnahmen bzw. zusätzliche Schmerzmittel einnehmen mussten.

Die Patienten haben den Fragebogen zunächst schriftlich bekommen und wurden dann telefonisch befragt, um auch auftretende Fragen direkt beantworten zu können. Die Patienten die keine telefonische Befragung wünschten, konnten den Fragebogen aber auch ausgefüllt an die Praxis zurücksenden. Selbstverständlich konnte die Befragung auch grundsätzlich abgelehnt werden. Die Ergebnisse der Befragungen wurden anschließend im Rahmen der vorliegenden Studie ausgewertet, nachdem die persönlichen Daten wie Name und Adresse geschwärzt wurden.

\subsection{Statistische Auswertung}

Die statistische Auswertung erfolgte mit einem Tabellenkalkulationsprogramm (Microsoft Office Excel 2003, Microsoft Corporation, Redmond, USA) und einem Statistikprogramm (StatSoft, Statsoft Inc., Tulsa, USA). Die Testung auf Normalverteilung der Daten erfolgte mit dem Kolmogorov-Smirnov-Test. Bei Annahme der Normalverteilung wurden ordinalskalierte Daten als Mittelwert und Standardabweichung angegeben. Kategoriale Daten wurden als prozentuale Anteile angegeben. Die Testung auf Unterschiede zwischen den Gruppen erfolgte für ordinalskalierte Daten mit dem Student-t-Test für unverbundene Stichproben und für kategoriale Daten mit dem Fisher-Exact-Test. Für alle Tests wurde die Irrtumswahrscheinlichkeit $\mathrm{p}<0,05$ als signifikant angenommen. 


\section{Ergebnisse}

\subsection{Patientendaten}

Es wurden insgesamt 83 Patienten in dieser Studie untersucht. Bei 41 Patienten wurde eine Allgemeinanästhesie durchgeführt (VN-Gruppe), bei 42 Patienten wurde zu der Allgemeinanästhesie außerdem eine interskalenäre Plexusanästhesie durchgeführt (VN+ISB-Gruppe). In Bezug auf Geschlecht, Alter, ASA-Klassifikation, Körpergröße, Körpergewicht und BMI waren beide Gruppen vergleichbar.

Tabelle 2: Allgemeine Patientendaten

\begin{tabular}{lccc}
\hline & $\begin{array}{c}\mathrm{VN} \\
\mathrm{n}=41\end{array}$ & $\begin{array}{c}\mathrm{VN}+\text { ISB } \\
\mathrm{n}=42\end{array}$ & $\mathrm{p}$-Wert \\
\hline männlich \% & 46 & 45 & 0,25 \\
Alter (Jahre) & $51 \pm 10$ & $53 \pm 9$ & 0,17 \\
ASA-Klassifikation (\%) & & & \\
I & 27 & 40 & 0,27 \\
II & 73 & 60 & 0,27 \\
Körpergröße (cm) & $170 \pm 7$ & $170 \pm 8$ & 0,25 \\
Body Mass Index $\left(\mathrm{kg} / \mathrm{m}^{2}\right)$ & $28 \pm 5$ & $28 \pm 5$ & 0,59 \\
\hline
\end{tabular}

Mittelwert \pm Standardabweichung

\subsection{Prozesszeiten}

Bei der Betrachtung der Prozesszeiten ergaben sich folgende Unterschiede zwischen den beiden Gruppen. Die Anlage der interskalenären Plexusblockade dauerte durchschnittlich 34,2 $( \pm 19,0)$ Minuten. Das bedeutet, dass auch die reine Anästhesiezeit (Zeit für Anlage der interskalenären Plexusanästhesie plus Anästhesie-kontrollierte Zeit) in der VN+ISB-Gruppe deutlich länger war. In der VN-Gruppe waren es 42,2 $( \pm 15,0)$ Minuten, während es in der VN+ISB-Gruppe 65,5 $( \pm 21,8)$ Minuten waren (p-Wert: <0,001). Die anästhesiologische Vorlaufzeit (Ankunft des Patienten im Operationssaal bis Freigabe Anästhesie) lag bei der VN-Gruppe bei durchschnittlich $31( \pm 21,4)$ Minuten. In der VN+ISB-Gruppe dagegen wurden durchschnittlich 18,1 $( \pm 6,0)$ Minuten benötigt. Damit war die Einleitung der Allgemeinanästhesie in der VN+ISB-Gruppe durchschnittlich 13 Minuten kürzer (p- 
Wert $<0,001$ ). Die Operationsdauer (Schnitt-Naht-Zeit) war in der VN+ISB-Gruppe kürzer. Die Eingriffe in Allgemeinanästhesie allein dauerten durchschnittlich 56,0 $( \pm 12,4)$ Minuten, die Operationen mit der Kombinationsanästhesie aus interskalenärer Plexusanästhesie und Allgemeinanästhesie dauerten nur 46,0 $( \pm 15,3)$ Minuten. Der p-Wert lag unter 0,002. Die anästhesiologische Nachlaufzeit (Ende nachbereitender operativer Maßnahmen bis Ende Präsenz Anästhesie-Arzt) war in beiden Gruppen gleich. In der VN-Gruppe lag sie bei 11,1 $( \pm 4,3)$ Minuten, in der VN+ISB-Gruppe lag sie bei 11,1 $( \pm 3,6)$ Minuten. Auch die „Anästhesie-kontrollierte Zeit“ (Anästhesiologische Vorlaufzeit plus anästhesiologische Nachlaufzeit) zeigte einen Vorteil für VN+ISB-Gruppe. In dieser Gruppe waren es 31,3 $( \pm 8,3)$ Minuten, während es in der VN-Gruppe 42,2 $( \pm 15)$ Minuten waren (p-Wert $<0,001)$.

Tabelle 3: Prozesszeiten

\begin{tabular}{lccc}
\hline & $\begin{array}{c}\mathrm{VN} \\
\mathrm{n}=41\end{array}$ & $\begin{array}{c}\mathrm{VN}+I S B \\
\mathrm{n}=42\end{array}$ & p-Wert \\
\hline Zeit für Anlage ISB (min) & k.A. & $34 \pm 19$ & k.A. \\
Anästhesiologischer Vorlauf (min) & $31 \pm 13$ & $18 \pm 6$ & $<0,001$ \\
Schnitt-Naht-Zeit (min) & $56 \pm 12$ & $46 \pm 15$ & $<0,002$ \\
Anästhesiologischer Nachlauf (min) & $11 \pm 4$ & $11 \pm 4$ & 0,71 \\
Anästhesie-kontrollierte Zeit (min) & $42 \pm 15$ & $31 \pm 8$ & $<0,001$ \\
Reine Anästhesiezeit (min) & $42 \pm 15$ & $66 \pm 22$ & $<0,001$ \\
\hline
\end{tabular}

Mittelwert \pm Standardabweichung

\subsection{Narkosemittelbedarf}

Bei allen Patienten wurde eine total-intravenöse Anästhesie (TIVA) durchgeführt. Es wurden neben Propofol, dass alle Patienten zur Narkoseaufrechterhaltung bekamen, auch Midazolam intravenös (v.a. präoperativ) zur Sedierung verwendet. Die Verwendung der Analgetika erfolgte nicht nach einem standardisiertem Vorgehen. So wurden neben Remifentanil und Sufentanil auch Alfentanil und S-Ketamin verwendet. 
In der VN-Gruppe bekamen 13 Patienten (31,7\%), in der VN+ISB-Gruppe 2 Patienten $(4,7 \%)$ zusätzlich S-Ketamin (p-Wert =0,002). Auch bei der Verwendung von Alfentanil ergaben sich signifikante Unterschiede. In der VN-Gruppe wurde bei 10 Patienten (24,4\%), in der VN+ISB-Gruppe bei 21 Patienten (50\%) Alfentanil verwendet $(\mathrm{p}$-Wert $=0,01)$. Die Verwendung von Midazolam zur Prämedikation war in beiden Gruppen vergleichbar. Midazolam wurde in der VN-Gruppe bei 37 Patienten (90,2\%) und bei 34 Patienten (81\%) in der VN+ISB-Gruppe verwendet $(\mathrm{p}$-Wert $=0,19)$. Der Bedarf an Propofol war bei beiden Gruppen vergleichbar. So bekam die VN-Gruppe durchschnittlich 7,6 mg/kg/h, während die VN+ISB-Gruppe einen Propofolbedarf von 7,7 mg/kg/h hatte (p-Wert: 0,7). Bei 34 Patienten der VN-Gruppe (82,9\%) wurde Remifentanil verwendet, in der VN+ISB-Gruppe waren es 33 Patienten (78,6\%). Bei der Betrachtung des Medians des Bedarfs waren beide Gruppen mit 0,08 $\mu \mathrm{g} / \mathrm{kgKG} / \mathrm{min}$ vergleichbar (p-Wert: 0,6). Sufentanil wurde in der VN-Gruppe bei 35 Patienten verwendet (85,3\%), während es in der VN +ISB-Gruppe 13 Patienten (31\%) waren. Auch hier war der Median des Bedarfs in beiden Gruppen mit $0,6 \mu \mathrm{g} / \mathrm{kg} / \mathrm{h}$ vergleichbar.

Tabelle 4: Narkosemittelbedarf

\begin{tabular}{lccc}
\hline & $\begin{array}{c}\text { VN } \\
\mathrm{n}=41\end{array}$ & $\begin{array}{c}\mathrm{VN}+\text { ISB } \\
\mathrm{n}=42\end{array}$ & $\mathrm{p}$-Wert \\
\hline Propofol $(\mathrm{mg} / \mathrm{kg} / \mathrm{h})$ & $7,6(6,2-9,7)$ & $7,7(5,1-9,8)$ & 0,7 \\
Remifentanil $(\%)$ & 83 & 79 & \\
Remifentanil $(\mu \mathrm{g} / \mathrm{kg} / \mathrm{min})$ & $0,08(0,05-0,11)$ & $0,08(0,04-0,12)$ & 0,6 \\
Sufentanil $(\%)$ & 85 & 31 & \\
Sufentanil $(\mu \mathrm{g} / \mathrm{kg} / \mathrm{h})$ & $0,60(0,49-0,72)$ & $0,60(0,42-0,73)$ & 0.9 \\
\hline \multicolumn{4}{l}{ Median $(25 .-75 . \%$ Percentile $)$}
\end{tabular}

\subsection{Interskalenäre Plexusanästhesie}

Die durchschnittliche Stimulationsgrenze der Nervenstimulation bei der Durchführung der interskalenären Plexusanästhesie lag bei $0,42 \mathrm{~mA}( \pm 0,11)$. Die Applikationsmenge von Mepivacain lag zwischen $25 \mathrm{ml}$ und $40 \mathrm{ml}$, durchschnittlich wurden $37,6 \mathrm{ml}( \pm 4,45)$ Mepivacain appliziert. 


\subsection{Hämodynamik}

Bei der Betrachtung der hämodynamischen Parameter wurde zunächst die Vormedikation der untersuchten Patienten betrachtet. In der VN-Gruppe nahmen 7 Patienten (17\%) dauerhaft Beta-Blocker ein, in der VN+ISB-Gruppe waren es 5 Patienten (11,9\%). Damit gab es keinen signifikanten Unterschied in beiden Gruppen (p-Wert: 0,55). Andere Antihypertensiva wurden sehr selten eingenommen. In beiden Gruppen nahmen lediglich 4 Patienten ACE-Hemmer ein und lediglich ein Patient in der VN+ISB-Gruppe nahm einen Calcium-Antagonisten ein.

Der dokumentierte durchschnittliche systolische Ausgangsblutdruck vor Lagerung in die beach-chair-Position lag bei der VN-Gruppe bei $132,32 \mathrm{mmHg}( \pm 15,58)$, in der VN+ISB-Gruppe bei 138,1 mmHg $( \pm 17,25)$. Der durchschnittliche systolische Blutdruck im Verlauf der gesamten Operation lag bei der VN-Gruppe bei $113,5 \mathrm{mmHg}( \pm 7,33)$ und in der VN+ISB-Gruppe bei $116,44 \mathrm{mmHg}( \pm 8,32)$. In diesen beiden Parametern zeigte sich zwischen beiden Gruppen kein Unterschied. Die p-Werte lagen bei 0,11 beim Ausgangsblutdruck und bei 0,09 beim durchschnittlichen Blutdruck.

Die dokumentierten durchschnittlichen maximalen und minimalen systolischen Blutdruckwerte lagen bei der VN-Gruppe bei 135,49 mmHg $( \pm 14,53)$ bzw. 96,34 $\operatorname{mmHg}( \pm 11,07)$, in der VN+ISB-Gruppe bei $146,43 \mathrm{mmHg}( \pm 19,01)$ bzw. 89,76 mmHg $( \pm 13,75)$. Damit war sowohl der maximale systolische Blutdruck bei der VN+ISB-Gruppe höher ( $\mathrm{p}$-Wert $<0,005$ ), als auch der minimale systolische Blutdruck niedriger ( $p$-Wert $<0,02)$.

Auch bei der Betrachtung des maximalen Abfalls des sytolischen Blutdrucks ergaben sich Unterschiede zwischen beiden Gruppen. So lag der durchschnittliche maximale Blutdruckabfall bei der VN-Gruppe bei 26,59\%, bei der VN+ISB-Gruppe bei $34,21 \%$ (p-Wert $<0,002)$.

Betrachtet man den Bedarf an Cafedrin und Theodrenalin - appliziert als KombiPräparat $\left(\right.$ Akrinor $^{\circledR}$ ) - zur Behandlung von therapiebedürftigen Hypotonien (definiert als MAD $<60 \mathrm{mmHg}$ bzw. Abfall des Blutdrucks unter 80\% des Ausgangs- 
blutdrucks), ergaben sich keine Unterschiede zwischen beiden Gruppen. In der VN-Gruppe war bei 27 Patienten (64\%) eine Akrinor ${ }^{\circledR}$-Gabe erforderlich, in der VN+ISB-Gruppe bei 31 Patienten (75\%) und es ergab sich kein signifikanter Unterschied (p-Wert: 0,45).

Bei der Betrachtung des gesamten perioperativ infundierten Volumens ergab sich kein Unterschied zwischen beiden Gruppen. In der VN-Gruppe wurden insgesamt $1448 \mathrm{ml}( \pm 209)$ Infusionslösung infundiert, in der VN+ISB-Gruppe waren es $1536 \mathrm{ml}( \pm 447)$. Jedoch wurden in der VN+ISB-Gruppe häufiger kolloidale Infusionen verwendet. In der VN/Gruppe bekamen 19 Patienten (46,3\%) kolloidale Infusionen, während in der VN+ISB-Gruppe 35 der Patienten (83,3\%) kolloidale Infusionen bekamen ( $\mathrm{p}$-Wert $<0,001)$.

Die dokumentierte durchschnittliche Ausgangs-Herzfrequenz lag bei der VNGruppe bei 77,63 $\mathrm{min}^{-1}( \pm 11,6)$, bei der VN+ISB-Gruppe bei $69,88 \mathrm{~min}^{-1}( \pm 12,8)$. Der p-Wert lag unter 0,01. Während der Operation lag die durchschnittliche Herzfrequenz in der VN-Gruppe bei $73,19 \mathrm{~min}^{-1}( \pm 8,3)$, bei der VN+ISB-Gruppe bei $67,38 \mathrm{~min}^{-1}( \pm 10,4)$. Auch hier lag der $\mathrm{p}$-Wert unter 0,01 . In der VN-Gruppe lag die maximale Herzfrequenz bei $83,88 \mathrm{~min}^{-1}( \pm 9,1)$, die minimale Herzfrequenz bei $66,25 \mathrm{~min}^{-1}( \pm 9,1)$. In der VN+ISB-Gruppe lag die maximale Herzfrequenz bei $77,86 \mathrm{~min}^{-1}( \pm 12,7)$, die minimale Herzfrequenz bei $60,36 \mathrm{~min}^{-1}( \pm 9,3)$. Im Vergleich beider Gruppen lag der p-Wert für die maximale Herzfrequenz bei unter 0,02, für die minimale Herzfrequenz unter 0,01. Damit waren alle gemessenen Herzfrequenzen in der VN-Gruppe höher. Die maximale Änderung der Herzfrequenz nach Lagerung in die beach-chair-Position war in beiden Gruppen vergleichbar. So stieg die Herzfrequenz in der VN-Gruppe um durchschnittlich 9,1\% $( \pm 10,8)$, während es in der VN+ISB-Gruppe eine Änderung von durchschnittlich 13,8\% ( \pm 23$)$ gab (p-Wert: 0,24). Bei einer therapiebedürftigen Bradykardie, definiert als Herzfrequenz unter $50 \mathrm{~min}^{-1}$, wurde Atropin appliziert. In der VN-Gruppe war bei 8 Patienten $(19,51 \%)$, in der VN+ISB-Gruppe bei 5 Patienten $(11,9 \%)$ eine Gabe notwendig. Damit ergab sich kein signifikanter Unterschied (p-Wert: 0,24). 
Tabelle 5: Hämodynamische Parameter

\begin{tabular}{|c|c|c|c|}
\hline & $\begin{array}{c}\text { VN } \\
n=41\end{array}$ & $\begin{array}{c}V N+I S B \\
n=42\end{array}$ & $\mathrm{p}$-Wert \\
\hline behandlungsbedürftige Hypotension (\%) & 66 & 74 & 0,48 \\
\hline behandlungsbedürftige Bradykardie (\%) & 20 & 12 & 0,30 \\
\hline infundiertes Volumen (ml) & $1448 \pm 209$ & $1536 \pm 447$ & 0,54 \\
\hline kolloidale Infusion \% & 46 & 83 & $<0,001$ \\
\hline \multicolumn{4}{|l|}{ systolischer Blutdruck (mmHg) } \\
\hline Rückenlage vor Narkoseeinleitung & $132 \pm 6$ & $138 \pm 17$ & 0,11 \\
\hline \multicolumn{4}{|l|}{ nach beach-chair-Position } \\
\hline Mittelwert & $114 \pm 7$ & $116 \pm 8$ & 0,09 \\
\hline Maximum & $135 \pm 15$ & $146 \pm 19$ & $<0,005$ \\
\hline Minimum & $96 \pm 11$ & $90 \pm 14$ & $<0,02$ \\
\hline maximaler Blutdruckabfall (\%) & -27 & -34 & $<0,002$ \\
\hline \multicolumn{4}{|l|}{ Herzfrequenz $\left(\min ^{-1}\right)$} \\
\hline Rückenlage vor Narkoseeinleitung & $78 \pm 12$ & $70 \pm 13$ & $<0,01$ \\
\hline \multicolumn{4}{|l|}{ nach beach-chair-Position } \\
\hline Mittelwert & $73 \pm 8$ & $67 \pm 10$ & $<0,01$ \\
\hline Maximum & $84 \pm 9$ & $78 \pm 13$ & $<0,01$ \\
\hline Minimum & $66 \pm 9$ & $60 \pm 9$ & $<0,02$ \\
\hline maximale Änderung der Herzfrequenz (\%) & $9 \pm 11$ & $14 \pm 23$ & 0,24 \\
\hline
\end{tabular}

Mittelwert \pm Standardabweichung

\subsection{Arterielle Sauerstoffsättigung}

Die dokumentierte durchschnittliche, pulsoxymetrisch gemessene arterielle Ausgangs-Sauerstoffsättigung lag bei der VN-Gruppe bei $99,34 \%( \pm 1,54)$, bei der VN +ISB-Gruppe, nach Anlage der interskalenären Plexusblockade, bei 98,62\% $( \pm 1,75)$. Der $p$-Wert lag unter 0,05 und war damit in der VN+ISB-Gruppe niedriger. Der Vergleich der maximalen und minimalen Sauerstoffsättigung ergab keinen Unterschied. In der VN-Gruppe war die maximale Sauerstoffsättigung 99,93\% $( \pm 0,26)$, die minimale Sauerstoffsättigung $99,17 \%( \pm 1,48)$. In der VN+ISB-Gruppe war die maximale Sauerstoffsättigung 99,71\% $( \pm 0,92)$, die minimale Sauerstoffsät- 
tigung 98,07\% ( $\pm 1,73)$. Für die maximale Sauerstoffsättigung ergab sich ein pWert von über 0,15, für die minimale Sauerstoffsättigung ergab sich ein p-Wert von über 0,77. Damit ergab sich kein Unterschied zwischen beiden Gruppen.

Neben den benötigten Analgetika, Hypnotika und Medikamente zur Therapie von Bradykardien und Hypotonien, wurden intraoperativ auch Metoclopramid (MCP) und Dexamethason appliziert. Die Indikation für diese Medikamente war die Prophylaxe der postoperativen Übelkeit, wobei das Dexamethason auch aus chirurgischer Indikation zur Prophylaxe einer postoperativen Schwellung gegeben wurde.

In beiden Gruppen bekamen jeweils 12 Patienten Dexamethason. In der VN-Gruppe entspricht das 29,27\%, in der VN+ISB-Gruppe 28,57\%. Der p-Wert liegt bei 0,94 und es ergab sich kein Unterschied. MCP bekamen in der VN-Gruppe 2 Patienten $(4,88 \%)$ in der VN+ISB-Gruppe nur ein Patient (2,38\%). Dort konnte ebenfalls kein signifikanter Unterschied festgestellt werden (p-Wert: 0,56).

\subsection{Patientenbefragung}

Von den 41 Patienten aus der VN-Gruppe konnten 36 Patienten (87,8\%) befragt werden. In der VN+ISB-Gruppe konnte bei 37 von 42 Patienten $(88,1 \%)$ die Befragung durchgeführt werden.

In der VN-Gruppe gaben 36 der Patienten (97\%) an, vor der Operation Schmerzen in der Schulter gehabt zu haben. In der VN+ISB-Gruppe waren es alle Patienten (100\%). Die Schmerzintensität auf der numerischen Rating-Skala (NRS) wurde bei der VN-Gruppe mit durchschnittlich 7,3 $( \pm 2,0)$ angegeben, in der VN+ISB-Gruppe mit durchschnittlich 7,4 $( \pm 1,5)$. Damit waren die präoperativen Schmerzen in beiden Gruppen vergleichbar ( $p$-Wert $>0,7)$. Auf die Frage ob vor der Operation regelmäßig Schmerzmittel eingenommen wurden, antworteten in der VN-Gruppe 23 der Patienten mit ,ja“ (64\%), in der VN+ISB-Gruppe nahmen 24 Patienten (64\%) regelmäßig Schmerzmittel ein.

Außerdem wurden die Patienten befragt, welche Analgetika vor der Operation eingenommen wurden. In der VN-Gruppe nahmen 22 Patienten nicht-steroidale Anti- 
rheumatika (NSAR) und 2 Patienten Tramadol ein. In der VN+ISB-Gruppe nahmen 23 Patienten NSAR und ein Patient nahm Metamizol als Analgetikum ein. Daraus ergab sich aus beiden Gruppen kein signifikanter Unterschied.

Postoperativ bekamen die Patienten Schmerzmittel verschrieben. Einmal ein NSAR (Ibuprofen $600 \mathrm{mg}$ ), welches sie fest über 7 Tage einnehmen sollten sowie für die ersten drei postoperativen Tage zusätzlich Metamizol $1 \mathrm{~g}$. Bei Bedarf, also bei starken Schmerzen, konnten die Patienten zusätzlich Tramadol einnehmen. Die Patienten wurden befragt, ob sie die Medikamente wie verschrieben eingenommen haben und ob sie von der Bedarfsmedikation Gebrauch machen mussten. In der VN-Gruppe haben 30 Patienten $(83, \%)$ die NSAR planmäßig eingenommen und 23 Patienten (64\%) haben auch die Bedarfsmedikation eingenommen. In der VN +ISB-Gruppe haben 31 Patienten (83,7\%) die NSAR planmäßig eingenommen und 18 Patienten (49\%) haben zusätzlich die Bedarfsmedikation eingenommen.

Damit gab es zwischen beiden Gruppen keinen Unterschied bei der Einnahme der festen Schmerzmittel-Medikation (p-Wert $>0,9$ ) und auch keinen Unterschied in der Einnahme der Bedarfsmedikation (p-Wert $>0,1)$.

Außerdem wurden die Patienten nach Schmerzen nach der Operation befragt und wenn sie Schmerzen hatten, wie stark diese waren (NRS). In der VN-Gruppe hatten 31 Patienten (86\%) am OP-Tag Schmerzen. Die gleiche Anzahl hatte auch am ersten und am zweiten Tag nach der Operation Schmerzen. In der VN+ISB-Gruppe hatten am OP-Tag 25 Patienten (68\%) Schmerzen. Am ersten Tag nach der Operation waren es 29 Patienten (78\%), am zweiten Tag nach der Operation 31 Patienten (84\%). Der p-Wert für die Schmerzen am OP-Tag war 0,06, am ersten Tag nach der Operation 0,39 und am zweiten Tag nach der Operation 0,78.

Betrachtet man die Schmerzintensität (NRS), ergab sich für die VN-Gruppe am Tag der Operation ein durchschnittlicher Wert von 4,11 $( \pm 2,55)$, am ersten Tag nach der Operation ein Wert von 3,77 $( \pm 2,43)$ und am zweiten Tag nach der Operation ein Wert von 3,2 $( \pm 2,27)$. In der VN+ISB-Gruppe war der durchschnittliche NRS-Wert am OP-Tag 2,67 $( \pm 2,58)$, am ersten Tag nach der Operation 2,7 $( \pm 2,27)$ 
und am zweiten Tag nach der Operation 2,67 $( \pm 2,24)$.

Damit hatten die Patienten in der VN+ISB-Gruppe am Tag der Operation signifikant weniger starke Schmerzen (p-Wert: 0,02). Am ersten Tag nach der Operation lag der p-Wert bei 0,06 und am zweiten Tag nach der Operation bei 0,33.

Des weiteren wurden die Patienten nach Problemen wie Übelkeit und Erbrechen, Shivering und bestehenden Missempfindungen im betroffenen Arm nach der Operation befragt. In der VN-Gruppe litten 8 Patienten (22\%) nach der Operation unter Übelkeit, 7 Patienten (18\%) mussten sich zudem übergeben. Bei einem Patienten (3\%) kam es zu einem postoperativen Shivering. In der VN+ISB-Gruppe kam es bei 4 Patienten (11\%) zu Übelkeit mit Erbrechen und bei 3 Patienten (8\%) zum postoperativen Shivering. Damit gab es keinen Unterschied zwischen beiden Gruppen in Bezug auf postoperative Übelkeit und Erbrechen (p-Wert: 0,19) oder postoperatives Shivering (p-Wert: 0,32).

Die Frage ob nach der Operation Missempfindungen im betroffenen Arm bestanden, beantworteten in der VN-Gruppe 4 Patienten mit ,ja“ (11\%), wobei in keinem Fall diese Beschwerden mehr als zwei Tage andauerten. In der VN+ISB-Gruppe bestanden ebenfalls bei 4 Patienten (11\%) postoperativ Missempfindungen, von denen 2 Patienten (5\%) diese Beschwerden länger als 2 Tage hatten. Es ergaben sich keine Unterschiede. Der p-Wert lag bei 0,97 bei den Missempfindungen, für die Beschwerden über 3 Tage hinaus ergab sich ein p-Wert von 0,15.

Abschließend wurden die Patienten befragt, wie sie den Erfolg der Operation in Bezug auf verbesserte Beweglichkeit im betroffenen Gelenk sowie Verringerung der Schmerzen einschätzen. In der VN-Gruppe gaben 29 Patienten (81\%) an, dass die Schmerzen nach dem Eingriff geringer waren. 28 Patienten (78\%) gaben an, dass sich die Beweglichkeit im Gelenk verbessert hat. In der VN+ISB-Gruppe gaben 26 Patienten (70\%) an, die Schmerzen seien nach der Operation geringer und 25 Patienten (68\%) beschrieben eine bessere Beweglichkeit im Schultergelenk. Damit wurde der Erfolg der Operation in beiden Gruppen vergleichbar eingeschätzt. In Bezug auf Verringerung der Schmerzen lag der p-Wert bei 0,31, in Be- 
zug auf die verbesserte Beweglichkeit lag der p-Wert bei 0,33.

Auf die Frage ob die Patienten das bei ihnen durchgeführte Narkoseverfahren weiterempfehlen würden, antworteten in der VN-Gruppe 33 Patienten (92\%) mit ,ja“,

Tabelle 6: Postoperative Ergebnisse

\begin{tabular}{|c|c|c|c|}
\hline & $\begin{array}{c}\mathrm{VN} \\
\mathrm{n}=36\end{array}$ & $\begin{array}{c}V N+I S B \\
n=37\end{array}$ & p-Wert \\
\hline \multicolumn{4}{|l|}{ präoperativer Schmerz } \\
\hline Patienten mit präoperativen Schmerzen (\%) & 97 & 100 & 0,31 \\
\hline präoperative Schmerzintensität (NRS) & $7 \pm 2$ & $7 \pm 2$ & 0,7 \\
\hline präoperativ Analgatikaeinnahme (\%) & 64 & 64 & 0,93 \\
\hline \multicolumn{4}{|l|}{ Inzidenz postoperativer Schmerz (\%) } \\
\hline Tag der Operation & 86 & 68 & 0,06 \\
\hline 1. postoperativer Tag & 86 & 78 & 0,39 \\
\hline 2. postoperativer Tag & 86 & 84 & 0,78 \\
\hline \multicolumn{4}{|l|}{ Intensität postoperativer Schmerz (NRS 1-10) } \\
\hline Tag der Operation & $4 \pm 3$ & $3 \pm 3$ & 0.02 \\
\hline 1. postoperativer Tag & $4 \pm 2$ & $3 \pm 2$ & 0,06 \\
\hline 2. postoperativer Tag & $3 \pm 2$ & $3 \pm 2$ & 0,33 \\
\hline \multicolumn{4}{|l|}{ intraoperativ verwendete Analgetika } \\
\hline S-Keatmin (\%) & 32 & 5 & 0,002 \\
\hline Midazolam (\%) & 90 & 81 & 0,19 \\
\hline Alfentanil (\%) & 24 & 50 & 0,01 \\
\hline \multicolumn{4}{|l|}{ postoperativer Schmerzmittelbedarf (\%) } \\
\hline Nicht-opioid Analgetika & 83 & 83 & 0,96 \\
\hline Opioidanalgetika & 64 & 49 & 0,19 \\
\hline \multicolumn{4}{|l|}{ postoperative Komplikationen (\%) } \\
\hline Übelkeit & 22 & 11 & 0,19 \\
\hline Erbrechen & 19 & 11 & 0,30 \\
\hline Shivering & 3 & 8 & 0,32 \\
\hline Parästhesie (> 2 Tage) & 0 & 0 & 0,97 \\
\hline \multicolumn{4}{|l|}{ Erfolg der Operation } \\
\hline Besserung des Schmerzes (\%) & 81 & 70 & 0,31 \\
\hline Beweglichkeit verbessert (\%) & 78 & 68 & 0,33 \\
\hline Zufriedenheit mit dem Narkoseverfahren (NRS 1-6) & $1,5 \pm 0,7$ & $1,5 \pm 0,9$ & 0,75 \\
\hline Würde Narkoseverfahren weiterempfehlen (\%) & 92 & 100 & 0,07 \\
\hline
\end{tabular}

Mittelwert \pm Standardabweichung 
in der VN+ISB-Gruppe waren es 37 Patienten (100\%). Der p-Wert lag bei 0,07.

Die Bewertung des Narkoseverfahrens durch Schulnoten $(1=$ sehr gut bis $6=$ ungenügend/schlecht) viel in der VN-Gruppe mit durchschnittlich 1,5 $( \pm 0,7)$ aus. Die VN+ISB-Gruppe bewertete ihr Verfahren ebenfalls mit 1,5 $( \pm 0,9)$. Der $p$-Wert lag bei 0,75 . Damit waren beide Narkoseverfahren in ihrer Akzeptanz vergleichbar und es ergab sich kein Unterschied. 


\section{Diskussion}

Die vorliegende Studie hat die interskalenäre Plexusanästhesie in Kombination mit einer total-intravenösen Anästhesie (TIVA) und die total-intravenöse Anästhesie allein für ambulante, arthroskopische Schulteroperationen verglichen. Betrachtet wurden Prozesszeiten, Narkosemittelbedarf, intraoperative Hämodynamik, postoperative Probleme wie PONV und Shivering, postoperative Schmerzen, Akzeptanz der Narkoseverfahren durch die Patienten sowie der Operationserfolg aus Sicht der Patienten.

\subsection{Bewertung der eigenen Ergebnisse}

Die Anlage der interskalenären Plexusanästhesie dauerte in der vorliegenden Studie 34,2 Minuten $( \pm 19,0)$. Diese Zeit ist vergleichbar mit bereits untersuchten Prozesszeiten von Brown et al. 1993 und D'Alessio et al. 1995. In der Studie von Brown et al. wurde der Zeitaufwand der Plexusanästhesie mit durchschnittlich 28 Minuten angegeben (Brown et al. 1993). D'Alessio et al. beschrieben eine durchschnittliche Zeit von 24 Minuten (D'Alessio et al. 1995a). Arciero et al. untersuchten die Dauer der Durchführung der interskalenären Plexusblockade bei unerfahrenem Personal. Für die ersten 50 Blockaden wurden bis zum OP-Beginn zunächst durchschnittlich 44 Minuten gebraucht. Bei den nächsten 50 Blockaden waren es dann nur noch 17 Minuten. Die Autoren machten damit auf die Wichtigkeit der Erfahrung des Anästhesisten aufmerksam, der die Plexus-Blockade durchführt (Arciero et al. 1996). Deswegen könnte auch ein Grund, warum so wenig niedergelassenen Anästhesisten die periphere Leitungsanästhesie verwenden, in mangelnder Erfahrung begründet sein. In der vorliegenden Studie wurden alle interskalenären Plexusblockaden von einem erfahrenem Facharzt durchgeführt.

Bei der Betrachtung der Schnitt-Naht-Zeit zeigte diese Studie, dass in der VN +ISB-Gruppe die Operationszeiten signifikant kürzer waren (46 vs. 56 Minuten). Ähnliche Ergebnisse zeigten auch Studien, bei dem Vergleich von Allgemeinanästhesie mit der interskalenären Plexusblockade allein. Bei Brown et al. (69 vs. 76 
Minuten), D'Alessio et al. (59 vs. 66 Minuten) und bei Arciero et al. (119 vs. 126 Minuten) waren die Schnitt-Naht-Zeiten in den ISB-Gruppen signifikant kürzer. D'Alessio et al. führten diese Ergebnisse auf verbesserte Operationsbedingungen durch einen niedrigeren Blutverlust und damit verbundener besseren arthroskopische Sicht zurück. Brown et al. und Arciero et al. gehen nicht weiter auf diese Ergebnisse ein. Daher ist die Annahme, dass die Operationsbedingungen bei einer Schulteroperation mit einer interskalenären Plexusanästhesie besser sind nur hypothetisch und es bedarf weiterer Untersuchungen (Brown et al. 1993, D'Alessio et al.1995a, Arciero et al. 1996).

Die Betrachtung des Anästhesiologischen Nachlaufs ergab in dieser Studie keinen Unterschied zwischen beiden Gruppen. In den o.g. Studien haben nur D'Alessio et al. auch die Prozesszeiten nach OP-Ende betrachtet und kamen zu dem Ergebnis, dass die Ausleitungszeit und die Zeit zum Umlagern bei den Patienten in der ISBGruppe deutlich kürzer waren als in der Allgemeinanästhesie-Gruppe. Auch Gonano et al. beschrieben eine kürzere Anästhesie-kontrollierte Zeit bei der Verwendung von interskalenärer Plexusanästhesie im Vergleich zur Allgemeinanästhesie (Gonano et al. 2009). Da in der vorliegenden Studie die ISB-Patienten zusätzlich eine Allgemeinanästhesie hatten sind die Ergebnisse mit dieser Studie nicht vergleichbar. Dass in der VN+ISB-Gruppe in der vorliegenden Studie die Anästhesie-Nachlauf-Zeit durch einen verringerten Opioid-Bedarf kürzer war konnte in dieser Studie nicht gezeigt werden.

Jedoch war in der VN+ISB die anästhesiologische Vorlaufzeit (Ankunft des Patienten im Operationssaal bis Freigabe Anästhesie) und damit auch die Anästhesiekontrollierte Zeit (anästhesiologische Vorlaufzeit plus anästhesiologische Nachlaufzeit) signifikant kürzer, während die reine Anästhesiezeit (Zeit für Anlage der interskalenären Plexusanästhesie plus Anästhesie-kontrollierte Zeit) in der VN +ISB-Gruppe natürlich deutlich länger war als in der VN-Gruppe. Daraus lässt sich schließen, dass wenn die Möglichkeit besteht die interskalenäre Plexusanästhesie in einem separaten Raum parallel zum Operationsbetrieb durchzuführen, die Pro- 
zesszeiten dadurch zu optimieren sind. Dafür sind allerdings ein zweiter Anästhesist und ein separater Raum zur Anlage der peripheren Plexusblockade notwendig. Gonano et al. verglichen die ökonomischen Aspekte der interskalenären Plexusblockade im Vergleich zur Allgemeinanästhesie und konnten zeigen, dass die Kosten bei einer interskalenären Plexusblockade günstiger waren als bei der Allgemeinanästhesie (Gonano et al. 2009). Zwar verglichen die Autoren die interskalenäre Plexusanästhesie allein und nicht, wie in der vorliegenden Studie, die Kombination aus beiden Verfahren mit der Allgemeinanästhesie, aber der Kostenvorteil ergab sich hauptsächlich aus den kürzeren Prozesszeiten bei dem Regionalanästhesieverfahren. Damit lässt sich annehmen, dass sich trotz der zusätzlichen Kosten für einen zweiten Anästhesisten durch die verbesserte Nutzung der OP-Kapazitäten auch bei der Kombinationsanästhesie ein ökonomischer Vorteil ergibt.

Alle Patienten in der vorliegenden Studie erhielten eine total-intravenöse Anästhesie (TIVA). Als Sedativum wurde bei allen Patienten Propofol verwendet und dieses mithilfe eines BIS-Monitors gesteuert und dosiert. Dabei ergab sich erwartungsgemäß kein Unterschied zwischen beiden Gruppen, und die Patienten erhielten mit 7,6 mg/kg/h in der VN-Gruppe bzw. $7,7 \mathrm{mg} / \mathrm{kg} / \mathrm{h}$ in der VN+ISB-Gruppe vergleichbare Mengen an Propofol. In der VN-Gruppe wurde bei 90,2\% der Patienten Midazolam verwendet in der VN+ISB-Gruppe bei 81\% der Patienten. Damit gab es keinen Unterschied zwischen beiden Gruppen.

Die Verwendung von Analgetika zur Allgemeinanästhesie war nicht standardisiert und die Anästhesisten konnten frei entscheiden, welche Analgetika sie verwendeten. Es wurden neben Remifentanil und Sufentanil auch Alfentanil und S-Ketamin in unterschiedlichen Kombinationen verwendet. Remifentanil wurde bei den meisten Patienten verwendet. In der VN-Gruppe wurde es bei $82,9 \%$, in der VN+ISBGruppe bei 78,6\% der Patienten verwendet. Wenn es verwendet wurde, war der mittlere Bedarf in beiden Gruppen vergleichbar und lag bei $0,08 \mu \mathrm{g} / \mathrm{kgKG} / \mathrm{min}$. Sufentanil wurde in der VN-Gruppe bei $85,4 \%$ der Patienten verwendet in der VN +ISB-Gruppe nur bei $31 \%$ der Patienten. Auch hier war bei Verwendung der mitt- 
lere Sufentanil-Bedarf in beiden Gruppen vergleichbar und lag bei $0,6 \mu \mathrm{g} / \mathrm{kgKG} / \mathrm{h}$. In der VN-Gruppe wurde bei 24,4\% der Patienten Alfentanil und bei 31,7\% S-Ketamin verwendet. In der VN+ISB-Gruppe wurde bei 50\% der Patienten Alfentanil und bei 4,7\% der Patienten S-Ketamin verwendet. Damit wurde in der VN-Gruppe häufiger S-Ketamin verwendet wurden, während in der VN+ISB-Gruppe häufiger Alfentanil verabreicht wurde.

Aus den o.g. Gründen ist eine Beurteilung des tatsächlichen intraoperativen Analgetika-Bedarfs nicht möglich. Auch ein Unterschied zwischen beiden Gruppen kann nicht festgestellt werden. Es erfolgte keine quantitative Messung des Analgetika-Bedarfs und die Applikation der Analgetika erfolgte nur nach klinischen Zeichen von Schmerzen bzw. war abhängig von den Erfahrungen des Anästhesisten, der die Narkose führte. Außerdem war der narkoseführende Anästhesist nicht verblindet und es war bekannt, ob der Patient eine interskalenäre Plexusanästhesie erhalten hatte oder nicht. So lässt sich die vermehrte Verwendung des kurzwirksamen Alfentanil in der VN+ISB-Gruppe am wahrscheinlichsten durch erwartete niedrige postoperative Schmerzen erklären. Die häufigere Verwendung von S-Ketamin in der VN-Gruppe lässt sich dagegen damit erklären, dass der Anästhesist höhere Dosen von Opioiden vermeiden wollte. Die Verwendung von Remifentanil und Sufentanil beruhte ebenfalls nur auf Erfahrungswerten des Anästhesisten und war damit nicht am tatsächlichen Bedarf orientiert. Außerdem kann bei einer relativ kurzen Operationsdauer eine standardisierte hohe Einleitungsdosis einen Unterschied im Verlauf der Operation verfälschen.

Trotz anderer Erwartung konnte daher kein Unterschied in beiden Gruppen in Bezug auf den intraoperativen Schmerzmittel-Bedarf gezeigt werden. Auch existieren lediglich nur zwei kleine Studien, die die Frage des intraoperativen Analgetika-Bedarfs bei Schulteroperationen bei Kombination aus Allgemein- und Regionalanästhesie behandelten. Brandl und Taegert verglichen 1991 den Fentanyl-Bedarf bei Schulteroperationen bei Patienten, die zu einer balancierten Allgemeinanästhesie eine interskalenären Plexusanästhesie bekamen, mit denen ohne zusätzlicher Re- 
gionalanästhesie. Sie zeigten einen niedrigeren Bedarf an Fentanyl, bei vergleichbaren Mengen von volatilen Anästhetika in der Gruppe mit Regionalanästhesie (Brandl und Taegert 1991). Grossi et al. konnten bei vergleichbaren Operationen einen niedrigeren Bedarf an Fentanyl bei gleicher Dosierung von Propofol, also im Rahmen einer TIVA zeigen (Grossi et al. 1998). Aber auch bei diesen Studien wurden lediglich klinische Zeichen verwendet um einen Analgetika-Bedarf zu erkennen. Ob eine Verblindung stattgefunden hat ist nicht beschrieben. Um den tatsächlichen intraoperativen Schmerzmittel-Bedarf zu bestimmen müsste daher eine verblindete Studie ggf. mit einer objektiven Messung der Schmerzintensität durchgeführt werden.

In beiden Gruppen gab es eine hohe Inzidenz für das Auftreten von behandlungsbedürftigen Hypotonien, nachdem die Patienten in die beach-chair-Position gebracht wurden. In der VN-Gruppe war dies bei 66\% der Patienten, in der VN+ISBGruppe bei $74 \%$ der Patienten der Fall. Einen signifikanten Unterschied gab es nicht und in beiden Gruppen haben eine vergleichbare Zahl von Patienten antihypertensive Medikamente bzw. Beta-Blocker eingenommen. In der VN+ISB-Gruppe kam es jedoch zu einem signifikant größerem Abfall des systolischen Blutdrucks, der minimale systolische Blutdruck war signifikant niedriger, und es wurde häufiger auf kolloidale Infusionslösungen zurückgegriffen. Außerdem waren sämtliche gemittelten Herzfrequenzen in der VN+ISB-Gruppe signifikant niedriger als in der VN-Gruppe.

Die hohe Inzidenz von behandlungsbedürftigen Hypotonien, wenn die Patienten in die beach-chair-Position verbracht werden, entspricht bereits publizierten Studien. So beobachteten Trentman et al. bei 54\% der Patienten ohne antihypertensive Dauermedikation eine behandlungsbedürftige Hypotension, bei Patienten mit antihypertensiver Dauermedikation sogar bei 75\% der Patienten. Kwak et al. beschreiben in $64 \%$ der Fälle eine behandlungsbedürftige Hypotension, bei Yadeau et al. kam es sogar bei 99\% der Patienten zu einer Hypotension (Kwak et al 2010, Yadeau et al. 2011). D'Alessio et al., Liguori et al. und Kahn und Hargett beschrieben das 
Auftreten von deutlichen Bradykardien mit Hypotonien (Bezold-Jarisch-Reflex) in der beach-chair-Position bei 13-28\% der Patienten, bei denen schulterchirurgische Eingriffe nur mit einer interskalenären Plexusblockade durchgeführt wurden (D'Alessio et al. 1995b, Liguori et al. 1998, Kahn und Hargett 1999). Im Gegensatz zu dieser Studie waren alle Patienten in diesen Studien wach und hatten keine zusätzliche Allgemeinanästhesie. Ozzeybek et al. betrachteten die Hämodynamik bei Patienten, die eine Kombination von interskalenärer Plexusanästhesie (mit Katheter) mit einer balancierten Allgemeinanästhesie zur Schulteroperation erhielten, im Vergleich zur interskalenäre Plexusanästhesie allein. Sie konnten zeigen, dass es bei der Kombination aus interskalenärer Plexusanästhesie und Allgemeinanästhesie zu vermehrten Blutdruckabfällen und Bradykardie kommt (Ozzeybek et al. 2003). In der vorliegenden Studie wurde erstmals ein Vergleich der Hämodynamik bei der total-intravenösen Anästhesie allein mit der Kombination aus total-intravenöser Anästhesie mit interskalenärer Plexusanästhesie im ambulanten Bereich angestellt. Der Vergleich mit den o.g. Studien ist nur eingeschränkt möglich, da unterschiedliche Patienten-Gruppen und unterschiedliche Narkoseverfahren (TIVA und balancierte Anästhesie) verglichen wurden. Dennoch scheint die beach-chair-Position besonders wichtig bei der Betrachtung der Hämodynamik zu sein und auch diese Studie zeigte ein hohe Inzidenz von behandlungsbedürftigen Hypotonien in beiden Patientengruppen. Ozzeybek et al. kamen nach ihrer Studie zu dem Schluss, dass die Durchführung einer Schulteroperation mit interskalenärer Plexusanästhesie allein zu favorisieren wäre, da die Kombination aus beiden Verfahren zu deutlicheren hämodynamischen Problemen führe (Ozzeybek et al. 2003). Demgegenüber stehen die Ergebnisse von D'Alessio et al., Liguori et al. und Kahn und Hargett, die das vermehrte Auftreten des Bezold-Jarisch-Reflexes bei wachen Patienten beschreiben (D'Alessio et al. 1995b, Liguori et al. 1998, Kahn und Hargett 1999). Die in dieser Studie festgestellten Unterschiede zwischen beiden Gruppen in Bezug auf den maximalen Blutdruckabfall, den niedrigeren minimalen Blutdruck und die häufigere Verwendung von kolloidalen Infusionslösungen in der VN-Gruppe sind 
nur eingeschränkt verwertbar. So war der maximale Blutdruckabfall in der VNISB-Gruppe von einem höheren Maximalblutdruck ausgehend und der geringere minimale systolische Blutdruck führte nicht zu einer häufigeren Verwendung von Vasopressoren. Außerdem war die Gesamtmenge von Infusionslösungen in beiden Gruppen vergleichbar. Auch konnte ein vermehrtes Auftreten von schwerer Bradykardie mit Hypotonie in der VN+ISB-Gruppe - im Sinne einer Aktivierung des Bezold-Jarisch-Reflexes - nicht gezeigt werden. Die durchschnittlichen Herzfrequenz-Werte in der VN+ISB-Gruppe waren zwar niedriger, aber in beiden Gruppen war selten eine Therapie mit Atropin notwendig und es konnte kein Unterschied gezeigt werden. Einschränkend muss erwähnt werden, dass die Herzfrequenz-Werte erst nach Anlage der interskalenären Plexusanästhesie dokumentiert wurden und so ein Effekt der Regionalanästhesie nicht dargestellt werden konnte. Außerdem wurde in der VN-Gruppe vermehrt S-Ketamin eingesetzt, das einen sympathomimetischen Effekt hat (Zielmann et al. 1997, Adams und Werner 1997). Das kann dazu geführt haben, dass es in der VN+ISB-Gruppe zu niedrigeren HerzfrequenzWerten und zu geringeren minimalen Blutdruck-Werten gekommen ist. Auch der geringere Blutdruckabfall in der VN-Gruppe kann durch die Verwendung von SKetamin erklärt werden.

In Bezug auf die Sauerstoffsättigung konnte in der VN+ISB-Gruppe eine niedrigere Sauerstoffsättigung vor der Einleitung der Allgemeinanästhesie festgestellt werden. Das kann an einer Betäubung des Nervus Phrenikus durch die interskalenäre Plexusblockade liegen, die mit einer Zwerchfell-Parese und damit mit einer eingeschränkten Vitalkapazität einhergehen kann (Urmey et al. 1991, Fujimura et al. 1995, Sala-Blanch et al. 1999, Hortense et al. 2010). Außerdem bekamen die Patienten bereits zur Anlage der interskalenären Plexusblockade eine Sedierung, welche die Sauerstoffsättigung negativ beeinflussen kann. Intraoperativ, also nach Intubation und während der Beatmung, gab es keine Unterschiede in beiden Gruppen.

Die Patientenbefragung ergab, dass in beiden Gruppen die Häufigkeit von Schmer- 
zen und die Schmerzintensität vor der Operation vergleichbar waren. Es ergaben sich keine Unterschiede darin, welche Schmerzmittel genommen wurden. Meistens wurden Nicht-Opioid-Analgetika eingenommen, zwei Patienten in der VN-Gruppe nahmen Tramadol ein.

Direkt nach der Operation hatten in der VN-Gruppe 86\% der Patienten Schmerzen, während in der VN+ISB-Gruppe 68\% der Patienten Schmerzen angaben. Der durchschnittliche NRS-Wert lag bei der VN-Gruppe bei 4,11, in der VN+ISBGruppe bei 2,67 und damit signifikant niedriger. $\mathrm{Zu}$ beachten ist dabei, das die VN-Gruppe mehr langwirksames Sufentanil bekommen hat, was die Schmerzen direkt nach der Operation positiv beeinflussen kann und damit den Unterschied zwischen beiden Gruppen verringert. Die positiven Effekte einer Plexusanästhesie innerhalb der ersten 24 Stunden nach einer Operation sind mehrfach beschrieben (Brown et al. 1993, Brand und Taegert 1991, Grossi et al. 1998, Gonano et al. 2009) und konnten mit dieser Studie bestätigt werden. Außerdem wurden in der VN+ISB-Gruppe vermehrt kurzwirksame Opioid-Analgetika wie Alfentanil und Remifentanil verwendet, was zu stärkeren postoperativen Schmerzen führen kann (Juckenhöfel et al. 1999, Grundmann et al. 1998). In dieser Studie konnte gezeigt werden, dass dieses Problem durch die Verwendung der interskalenären Plexusanästhesie verhindert werden kann. Im Verlauf der ersten beiden postoperativen Tage konnte in den beiden Gruppen kein Unterschied zwischen Schmerzintensität und dem Schmerzmittelbedarf festgestellt werden. Trompeter et al. untersuchten 2010 bereits, wie sich die Schmerzen der Patienten in den ersten 5 Tagen nach einer arthroskopischen Schulteroperation entwickelten, um die Frage zu klären, ob ein Katheterverfahren für solche Eingriffe sinnvoll wäre und ob es nach Abklingen der Plexusanästhesie zu vermehrten Schmerzen kommen würde. Sie kamen zu dem Ergebnis, dass eine single-shot-Blockade ausreichend sei, da die Patienten postoperativ nur einfache Analgetika benötigten und die Zufriedenheit der Patienten sehr hoch war. Einen Vergleich mit Patienten, die nur eine Allgemeinanästhesie bekamen stellten die Autoren jedoch nicht an (Trompeter et al. 2010). In dieser Studie 
ist eine Tendenz zu erkennen, dass die interskalenäre Plexusanästhesie auch nach Entlassung nach Hause positive Effekte zeigt. Ein vermehrtes Auftreten von starken Schmerzen nach Abklingen der Regionalanästhesie konnte nicht festgestellt werden.

Die Patienten wurden nach Problemen nach der Operation bzw. der Narkose gefragt. Die Patienten in der VN+ISB-Gruppe hatten zu 11\% Probleme mit postoperativer Übelkeit, während es in der Gruppe mit Allgemeinanästhesie allein 22\% der Patienten waren. Intraoperativ bekamen beide Gruppen vergleichbare Mengen an prophylaktischen Medikamenten wie Dexamethason und Metoclopramid und somit waren beide Gruppen vergleichbar. Das es nach Regionalanästhesieverfahren allein seltener zu postoperativer Übelkeit kommt, ist mehrfach beschrieben (Brown et al 1993, D'Alessio et al 1995a, Liu et al. 2005). Außerdem kann durch die Verwendung von Propofol und das Verzichten auf volatile Anästhetika, also der Durchführung einer total-intravenösen Anästhesie (TIVA), die Inzidenz von postoperativer Übelkeit und Erbrechen deutlich gesenkt werden (Borgeat und Stirnemann 1998). Dass eine Kombination aus Regionalanästhesie mit der TIVA diese positiven Effekte verstärkt, ist noch nicht beschrieben und konnte auch in dieser Studie nicht gezeigt werden. Weitere Studien wären nötig, um diese Frage zu beantworten.

Die Frage nach postoperativem Kältezittern beantwortete ein Patienten (3\%) der VN-Gruppe mit ,ja“, in der VN+ISB-Gruppe waren es 3 Patienten (8\%) und es ergab sich kein Unterschied zwischen beiden Gruppen. Das es bei der Durchführung einer totalintravenösen Anästhesie im Vergleich zur Allgemeinanästhesie mit volatilen Anästhetika vermehrt zum Shivering kommt, ist bereits beschrieben worden (Hogue et al.1996, Juckenhöfel et al. 1999, Grundmann et al. 1998). In dieser Studie haben beide Patienten-Gruppen eine TIVA erhalten. Dass die zusätzliche interskalenäre Plexusanästhesie hierauf einen Effekt hat, ist nicht zu erkennen und weitere Studien zu dem Thema fehlen.

Eine explizite Untersuchung von Nebenwirkungen der interskalenären Plexusanäs- 
thesie wie Horner-Syndrom, Phrenikus- oder Recurrens-Parese erfolgte nicht. In der Praxis werden aber schwerwiegende Komplikationen dokumentiert wenn Sie auftreten. Bei keinem der Patienten in dem untersuchten Kollektiv wurde eine schwere Komplikation beschrieben. Auch bei der Befragung hat kein Patient über Symptome wie Heiserkeit oder hängendes Augenlid o.ä. geklagt. Eine Aussage zur Inzidenz von Horner-Syndrom, Phrenikus-Parese oder Recurrens-Parese in dieser Studie lässt sich dennoch nicht treffen. Es ist aber anzunehmen, dass keine schweren oder langfristigen Probleme nach der interskalenären Plexusanästhesie aufgetreten sind. In Bezug auf Missempfindungen nach der Operation beschrieben in beiden Gruppen jeweils 4 Patienten (11\%) solche Parästhesien. Eine Unterscheidung, ob diese Missempfindungen operations- bzw. lagerungsbedingt waren oder als Folge der interskalenären Plexusblockade zu sehen sind, kann nur schwer erfolgen. Jedoch kam es in der VN+ISB-Gruppe nicht zu einem vermehrten Auftreten. Die Inzidenz von vorübergehendem neurologischen Defizit nach Regionalanästhesie-Verfahren wird mit 2,3-2,84\% der Fälle beschrieben (Bishop et al. 2005, Brull et al. 2007). Nervenläsionen oder permanente Nervenschäden nach Regionalanästhesie sind sehr selten, und werden mit 0,02-0,04\% der Fälle beschrieben (Barrington et al. 2009, Auroy et al. 2002, Auroy et al 1997, Capdevila und al. 2005, Brull et al. 2007, Bishop et al. 2005). Aufgrund dieser geringen Inzidenz sind größere Fallzahlen als in dieser Studie nötig, um valide Aussagen zum Auftreten dieser Komplikationen zu machen.

Die Patienten sind nach ihrer Zufriedenheit mit dem Narkoseverfahren und nach der Beurteilung des Erfolges der Operation befragt worden. Unterschiede in der Patientenzufriedenheit bei der Bewertung mittels Schulnoten konnten in dieser Studie nicht festgestellt werden. In der VN+ISB-Gruppe gaben alle Patienten an, dass Sie dieses Narkoseverfahren weiterempfehlen würden. In der VN-Gruppe waren es 92\%. Damit gab es keinen Unterschied zwischen beiden Gruppen und man kann eine hohe Akzeptanz der Patienten in Bezug auf die interskalenäre Plexusblockade erkennen. In der Studie von Ozzeybek et al. konnte kein Unterschied in der 
Akzeptanz der Patienten zwischen interskalenärer Plexusanästhesie und der Kombinationsanästhesie festgestellt werden, wobei dort beide Patienten-Gruppen eine interskalenäre Plexusanästhesie erhielten (Ozzeybek et al. 2003). Damit scheint die gegebenenfalls unangenehme Prozedur der Anlage der interskalenären Plexusanästhesie keinen negativen Einfluss auf die Patientenzufriedenheit zu haben. Die Studien von Shevade und Panagopoulos und von Matthey et al. beschreiben, dass die meisten Patienten grundsätzlich eine Allgemeinanästhesie bevorzugen, da sie von der Operation nichts mitbekommen möchten (Shevade und Panagopoulos 1991, Matthey et al. 2004). Diese Annahme beschreiben auch Grossi et al., ohne jedoch wissenschaftliche Ergebnisse dazu zu liefern (Grossi et al. 1998).

Bei der Beurteilung des Erfolges der Operation seitens der Patienten gab es keine Unterschiede. Der größte Teil der Patienten stellte eine Verbesserung der Gelenkbeweglichkeit und der Schmerzen nach der Arthroskopie fest. Der Erfolg einer Operation ist entscheidend von der Diagnose abhängig, und ein Narkoseverfahren kann den Operationserfolg nur schwer beeinflussen. Die Kombination aus Allgemeinanästhesie und interskalenärer Plexusblockade scheint nach dieser Studie weder positiven noch negativen Einfluss auf den Erfolg der Operation zu haben.

\subsection{Methodenkritik und Fehlermöglichkeiten}

In Bezug auf allgemeine Patientendaten wie Alter, Geschlecht, Körpergewicht und Körpergröße sowie ASA-Klassifikation waren beide Patientengruppen vergleichbar. Die Entscheidung, ob ein Patient eine interskalenäre Plexusanästhesie zu der Allgemeinanästhesie erhielt, hat der zuständige Anästhesist selbstständig nach medizinischen und organisatorischen Kriterien getroffen. Vorteile ergaben sich daraus, dass die Plexusanästhesie immer von einem erfahrenen Facharzt für Anästhesiologie durchgeführt wurde. In dieser Studie wurde die interskalenäre Plexusanästhesie mittels Nervenstimulation durchgeführt und nicht mithilfe von Ultraschall. Die aktuelle Studienlage beschreibt aber deutliche Vorteile der Sonographie in Bezug auf Blockadeerfolg, Dauer der Anlage sowie Anschlagzeit und Dauer der Blockade. 
Außerdem scheint auch die Komplikationsrate niedriger zu sein, wenn die Regionalanästhesie mit Ultraschall durchgeführt wird (Abrahams et al. 2009, Griffin und Nicholls 2010).

Ein weiterer Nachteil der vorliegenden Studie ist die Kombination verschiedener Analgetika zur Allgemeinanästhesie, was eine Aussage über den intraoperativen Schmerzmittelbedarf erschwert.

Die Patientenbefragung ist nicht direkt nach der Operation erfolgt und damit mussten die Patienten teilweise aus ihrer Erinnerung antworten. Die aus Befragungen hervorgegangenen Daten sind immer subjektiv und bedürfen eines genauen Verständnisses der Fragen. Die Patienten hatten den Fragebogen schriftlich bekommen, konnten sich ausreichend lange damit beschäftigen und wurden dann telefonisch befragt. Das ermöglichte den Patienten genauer nachzufragen, wenn Fragen nicht richtig verstanden wurden und zusätzliche Anmerkungen zu machen, die im Fragebogen nicht erfasst wurden. 


\section{Zusammenfassung}

Diese Studie konnte zeigen, dass die Kombination einer totalintravenösen Anästhesie mit einer interskalenären Plexusanästhesie die postoperativen Schmerzen am Tag des Eingriffs positiv beeinflusst. Auch bei der Verwendung von kurzwirksamen Opioid-Analgetika kommt es nach der Operation nicht zu stärkeren Schmerzen, und die Schmerzen nach Abklingen der Regionalanästhesie sind nicht verstärkt. Ein positiver Effekt der Kombination aus peripherer Leitungsanästhesie und totalintravenöser Anästhesie auf postoperative Übelkeit und Erbrechen konnte nicht gezeigt werden. Wenn Patienten zur Schulterarthroskopie in die halb-sitzende beach-chair-Position gebracht werden, muss grundsätzlich mit einem Blutdruckabfall gerechnet werden. Die Kombination aus interskalenärer Plexusanästhesie und totalintravenöser Anästhesie führt bei ASA I- und ASA II-Patienten nicht zu einem vermehrten Auftreten von behandlungsbedürftigen Hypotonien oder zu einem vermehrten Auftreten des Bezold-Jarisch-Reflexes mit schwerer Bradykardie und kann somit auch im ambulanten Bereich mit einer hohen Patientensicherheit eingesetzt werden. Die Patientenzufriedenheit ist bei beiden Narkoseverfahren groß. Die potentiell unangenehme Prozedur der Anlage der interskalenären Plexusanästhesie hat keinen negativen Einfluss auf die Patientenzufriedenheit. Schwere Komplikationen bei der interskalenären Plexusanästhesie, wie permanente Nervenläsionen sind sehr selten. Damit kann dieses Regionalanästhesie-Verfahren grundsätzlich auch im ambulanten Bereich eingesetzt werden. Die Kombination aus Regionalanästhesie und Allgemeinanästhesie hat einen positiven Effekt auf die operativen Prozesszeiten, wenn räumliche und personelle Gegebenheiten geschaffen sind. AuBerdem scheint die Operationszeit mit interskalenärer Plexusanästhesie kürzer zu sein. Die Gründe dafür sind aber unklar, und es bedarf weiterer Studien. Das operative Ergebnis scheint nicht durch das Narkoseverfahren beeinflusst. 


\section{Anhang}

\subsection{Fragebogen zur Patientenzufriedenheit}

\section{Vor dem Eingriff}

1.1 Hatten Sie vor dem operativen Eingriff Schmerzen?

\section{Ja $\bigcirc$ Nein $\bigcirc$}

\subsubsection{Wenn ja wo?}

1.1.2 Wie stark würden Sie die Schmerzen beschreiben? (Markieren Sie die Stärke des Schmerzes, wobei der Wert ,0' kein Schmerz und ,10' der stärkste vorstellbare Schmerz sei)

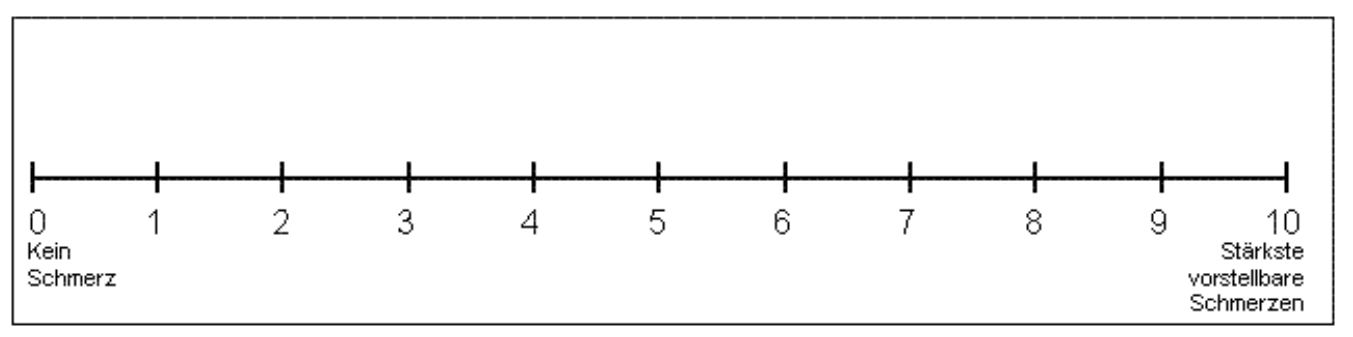

1.2 Haben Sie vor dem Eingriff regelmäßig Schmerzmittel eingenommen?

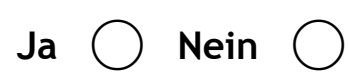

1.2.1 Wenn ja, welche?

1.2.2 Wie lange haben Sie die Schmerzmedikamente eingenommen?

1.3 Wurde bei Ihnen bereits dieses oder ein anderes Verfahren der Regionalanästhesie alleine, oder als Ersatz für eine Vollnarkose durchgeführt (zum Beispiel Rückenmarksnarkose)?

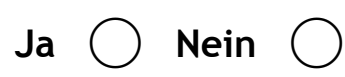

\section{Während des Eingriffes}

2.1 Haben Sie während des operativen Eingriffes Schmerzen verspürt?

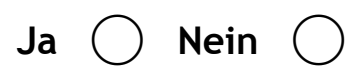

2.1.1 Wenn ja, wie stark waren diese Schmerzen? (Markieren Sie die Stärke des Schmerzes, wobei der Wert ,0`kein Schmerz und ,10' der stärkste vorstellbare Schmerz sei)

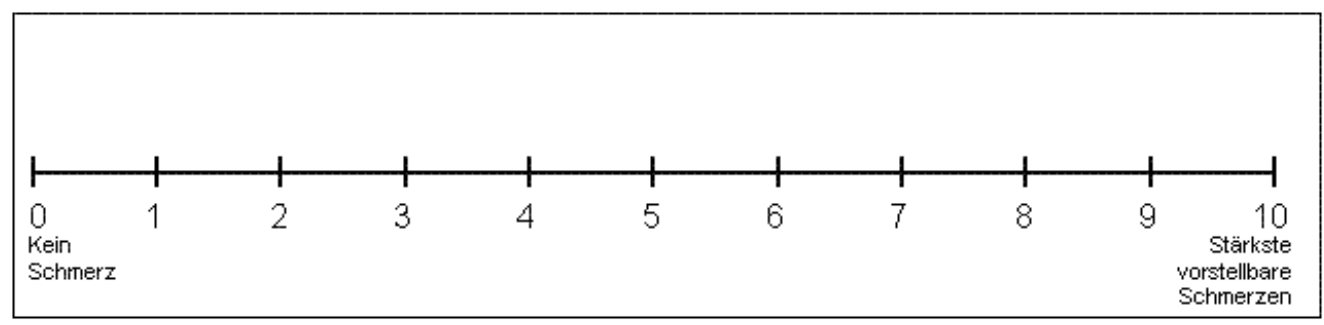




\section{Nach dem Eingriff}

3.1 Wir haben Ihnen für die Zeit nach der Operation Schmerzmedikamente verordnet/ mitgegeben. Dies umfasste Ibuprofen oder Diclofenac, sowie bei Bedarf Novamin und/oder Tramadol.

Haben Sie die mitgegebenen Schmerzmedikamente nach Plan eingenommen?

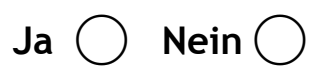

3.1.1 Wenn nicht, welche Schmerzmittel haben Sie nach dem Eingriff eingenommen?

3.2 Haben Sie die bei Schmerzen vorgesehene Bedarfsmedikation (Tramal Tropfen) eingenommen?

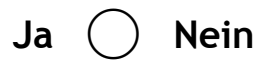

\section{Diese Fragen betreffen Schmerzen nach dem Eingriff:}

\subsection{Haben Sie direkt nach dem Eingriff oder noch am selben Tag des Eingriffes}

Schmerzen verspürt?

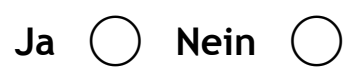

3.3.1 Wenn ja, wie stark waren diese Schmerzen? (Markieren Sie die Stärke des Schmerzes, wobei der Wert ,0‘ kein Schmerz und ,10‘ der stärkste vorstellbare Schmerz sei)

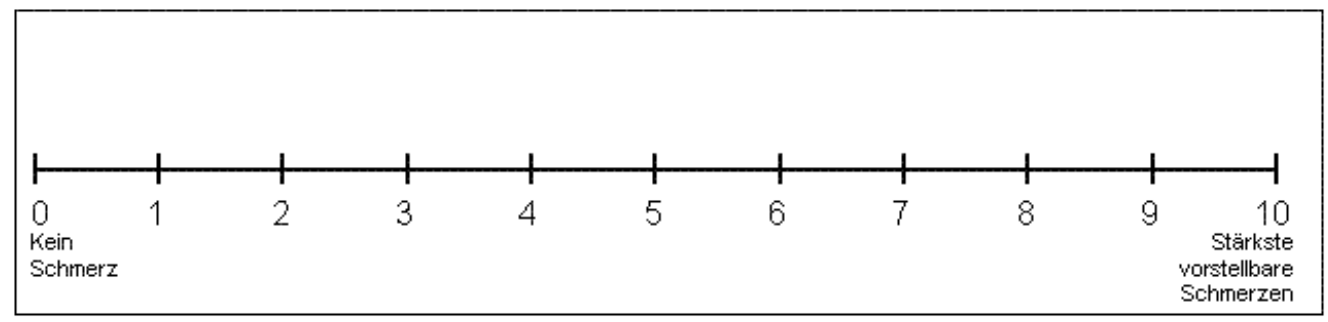

3.4 Haben Sie am 1. Tag nach dem Eingriff Schmerzen verspürt?

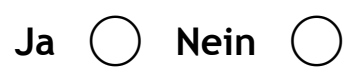

3.4.1 Wenn ja, wie stark waren diese Schmerzen? (Markieren Sie die Stärke des Schmerzes, wobei der Wert ,0‘ kein Schmerz und ,10' der stärkste vorstellbare Schmerz sei)

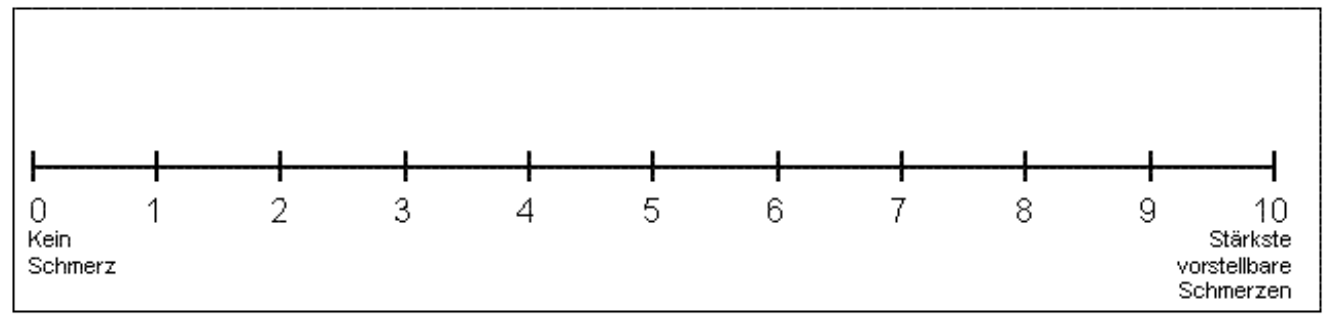


3.5 Haben Sie am 2. Tag nach dem Eingriff Schmerzen verspürt?

\section{Ja $\bigcirc$ Nein $\bigcirc$}

3.5.1 Wenn ja, wie stark waren diese Schmerzen? (Markieren Sie die Stärke des Schmerzes, wobei der Wert , 0 ‘ kein Schmerz und ,10` der stärkste vorstellbare Schmerz sei)

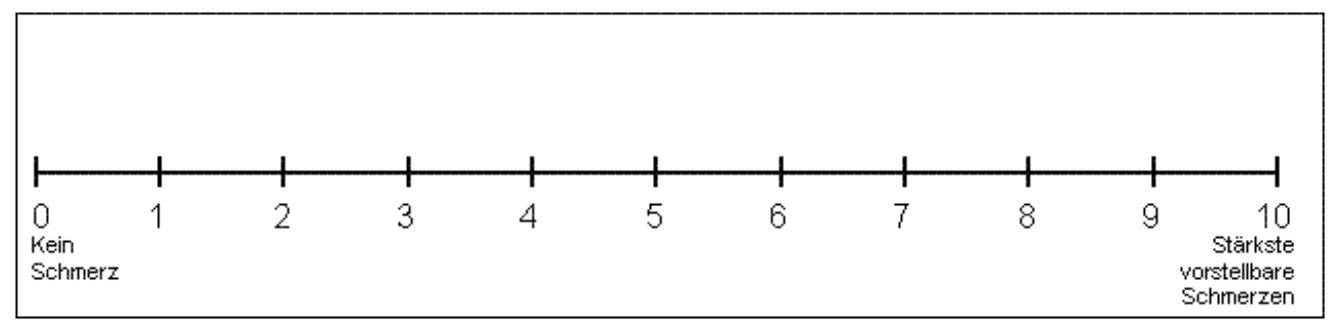

Diese Fragen betreffen Auffälligkeiten nach dem Eingriff:

3.6 Verspürten Sie direkt nach dem operativen Eingriff oder am Folgetag Übelkeit?

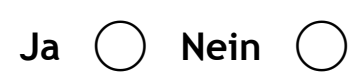

3.7 Mussten Sie sich direkt nach dem operativen Eingriff oder am Folgetag brechen?

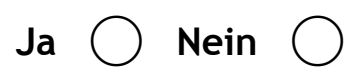

3.7.1 Wenn ja, wie oft?

1-2 Mal $\bigcirc$ Häufiger

3.8 Litten Sie direkt nach dem Eingriff unter Zittern?

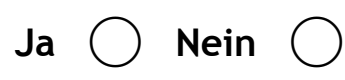

3.9 Verspürten Sie ab dem 1. Tag nach dem Eingriff noch Missempfindungen, wie Kribbeln, Taubheitsgefühle oder Lähmungen an dem operierten Arm/Schulter?

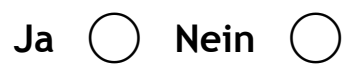

3.9.1 Wenn ja, wie lange haben diese Missempfindungen angehalten

3.9.2 Wenn ja, welche Missempfindungen haben Sie wahrgenommen? 


\section{Zufriedenheit}

4.1 Waren Sie mit dem Verfahren (Regionalanästhesie oder Allgemeinanästhesie) zufrieden? ( Benotung nach Schulnoten )

Schulnote $(1-6)$ :

4.2 Würden Sie einem Bekannten dieses Verfahren der (Regionalanästhesie oder Allgemeinanästhesie) weiterempfehlen?

Ja $\bigcirc$ Nein $\bigcirc$

4.3 Hat sich die Schmerzhaftigkeit des Gelenkes durch den Eingriff verringert?

Ja $\bigcirc$ Nein $\bigcirc$

4.4 Hat sich die Beweglichkeit des Gelenkes durch den Eingriff verbessert?

Ja $\bigcirc$ Nein 


\subsection{Tabellen- und Abbildungsverzeichnis}

Tabelle 1: Kontraindikationen zur Anlage peripherer Nervenblockaden 10

Tabelle 2: Allgemeine Patientendaten__ 29

Tabelle 3: Prozesszeiten__ 30

Tabelle 4: Narkosemittelbedarf___ 31

Tabelle 5: Hämodynamische Parameter___ 34

Tabelle 6: Postoperative Ergebnisse _ 38

Abbildung 1: Plexus brachialis___ 14

Abbildung 2: Punktionsstelle zur interskalenären Plexusanästhesie___ 21

Abbildung 3: Durchführung einer interskalenären Plexusanästhesie___ 23

Abbildung 4: BIS VISTA Monitor ${ }^{\circledR}$

Abbildung 5: Platzierung der BIS-Quatro-Elekroden ${ }^{\circledR} \_25$ 


\section{Literaturverzeichnis}

Abrahams MS, Aziz MF, Fu RF, Horn JL (2009): Ultrasound guidance compared with electrical neurostimulation for peripheral nerve block: a systematic review and meta-analysis of randomized controlled trials. Br J Anaesth $\underline{102}$, 408-417

Adams HA, Werner C (1997): Vom Razemat zum Eutomer: (S)-Ketamin, Renaissance einer Substanz? Anaesthesist $\underline{46}$, 1026-1042

Apfel CC, Roewer N (2000): Einflussfaktoren von Übelkeit und Erbrechen nach Narkosen Fiktionen und Fakten. Anaesthesist 499, 629-642

Apfel CC, Greim C-A, Goepfert C, Grundt D, Usadel J, Sefrin P, Roewer N (1998): Postoperatives Erbrechen Ein Score zur Voraussage des Erbrechensrisikos nach Inhalationsanaesthesien. Anaesthesist 47, 732-740

Arciero RA, Taylor DC, Harrison SA, Snyder RJ, Leahy KE, Uhorchak JM (1996): Interscalene anesthesia for shoulder arthroscopy in a community-sized military hospital. Arthroscopy 12, 715-719

Auroy Y, Narchi P, Messiah A, Litt L, Rouvier B, Samii K (1997): Serious complications related to regional anesthesia: results of a prospective survey in France. Anesthesiology $\underline{87}, 479-486$.

Auroy Y, Benhamou D, Bargues L, Ecoffey C, Falissard B, Mercier F, Bouaziz H, Samii K (2002): Major complications of regional anesthesia in France.

Anesthesiology 97, 1274-1280

Barrington MJ, Watts SA, Gledhill SR, Thomas RD, Said SA, Snyder GL, Tay VS, Jamrozik K (2009): Preliminary results of the australasian regional anaesthesia collaboration. Reg Anesth Pain Med 34, 534-541

Bauer M, Diemer M, Ansorg J, Schleppers A, Bauer K, Bomplitz M, Tsekos E, Hanss R, Schuster M (2008): Glossar perioperativer Prozesszeiten und Kennzahlen - Eine gemeinsame Empfehlung von DGAI, BDA, BDC und VOPM. Anästh Intensivmed $\underline{49}$, S93-S105

Benumof JL (2000): Permanent loss of cervical spinal cord function associated with interscalene block performed under general anesthesia. Anesthesiology $\underline{93}$, $1541-1544$

Birnbaum J, Klotz E, Bogusch G, Volk T (2007): Elektrische Nervenstimulation für Plexus- und Nervenblockaden. Anaesthesist $\underline{56}, 1155-1162$ 
Bishop JY, Sprague M, Gelber J, Krol M, Rosenblatt MA, Gladstone J, Flatow EL (2005): Interscalene regional Aanesthesia for shoulder surgery. J Bone Joint Surg Am $\underline{87}, 974-979$

Borgeat A, Stirnemann HR (1998): Antiemetische Wirkung von Propofol. Anaesthesist 47, 918-924

Borgeat A, Ekatodramis G, Kalberer F, Benz C. (2001): Acute and nonacute complications associated with interscalene block and shoulder surgery: a prospective study. Anesthesiology $\underline{95}, 875-880$

Brandl F, Taeger K (1991): The combination of general anesthesia and interscalene block in shoulder surgery. Anaesthesist $\underline{40}, 537-542$

Brown AR, Weiss R, Greenberg C, Flatow EL, Bigliani LU (1993): Interscalene block for shoulder arthroscopy: comparison with general anesthesia. Arthroscopy $\underline{9}, 295-300$

Brull R, McCartney CJL, Chan VWS, El-Beheiry H (2007): Neurological complications after regional anesthesia: contemporary estimates of risk. Anesth Analg 104, 965-974

Campagna JA, Carter C (2003): Clinical relevance of the Bezold-Jarisch reflex. Anesthesiology 98, 1250-1260

Capdevila X, Pirat P, Bringuier S, Gaertner E, Singelyn F, Bernard N, Choquet O, Bouaziz H, Bonnet F (2005): Continuous peripheral nerve blocks in hospital wards after orthopedic surgery. Anesthesiology 103, 1035-1045

Chan VW, Peng PW, Kaszas Z, Middleton WJ, Muni R, Anastakis DG, Graham BA (2001): A comparative study of general anesthesia, intravenous regional anesthesia, and axillary block for outpatient hand surgery: clinical outcome and cost analysis. Anesth Analg 93, 1181-1184.

Chapman GM (1972): Regional nerve block with the aid of a nerve stimulator. Anaesthesia 27, 185-193

D'Alessio JG, Rosenblum M, Shea KP, Freitas D (1995a): A retrospective comparison of interscalene block and general anesthesia for ambulatory surgery shoulder arthroscopy. Reg Anesth Pain Med 20, 62-68 
D'Alessio JG, Weller RS, Rosenblum M (1995b): Activation of the Bezold-Jarisch reflex in the sitting position for shoulder arthroscopy using interscalene block. Anesth Analg $\underline{80}, 1158-1162$

De Andrés J, Sala-Blanch X (2001): Peripheral nerve stimulation in the practice of brachial plexus anesthesia: a review. Reg Anesth Pain Med 26, 478-483

Deutsche Interdisziplinäre Vereinigung für Schmerztherapie (2008): S3-Leitlinie, Behandlung akuter perioperativer und posttraumatischer Schmerzen. Deutscher Ärzte-Verlag, Köln und AWMF-Reg.-Nr. 041/001

DGAI Deutsche Gesellschaft für Anästhesiologie und Intensivmedizin/BDA Berufsverband deutscher Anästhesisten (2006): Vereinbarung zur Qualitätssicherung ambulante Anästhesie des Berufsverbandes Deutscher Anästhesisten, der Deutschen Gesellschaft für Anästhesiologie und Intensivmedizin und des Berufsverbandes der Deutschen Chirurgen. Anästh Intensivmed $\underline{47}, 50-51$

Dullenkopf A, Borgeat A (2003): Lokalanästhetika - Unterschiede und Gemeinsamkeiten der „,caine“. Anaesthesist 52, 329-340

Eikaas H, Reader J (2009): Total intravenous anaesthesia techniques for ambulatory surgery. Curr Opin Anaesthesiol 22, 725-729

Frank SM, Beattie C, Christopherson R, Norris EJ, Perler BA, Williams GM, Gottlieb SO (1993): Unintentional hypothermia is assoziated with postoperative myocardial ischemia. Anesthesiology $\underline{78}, 468-476$

Fujimura N, Namba H, Tsunoda K, Kawamata T, Taki K, Igarasi M, Namiki A (1995): Effect of hemidiaphragmatic paresis caused by interscalene brachial plexus block on breathing pattern, chest wall mechanics, and arterial blood gases. Anesth Analg $\underline{81}, 962-966$

Gan TJ, Meyer TA, Apfel CC, Chung F, Davis PJ, Habib AS, Hooper VD, Kovac AL, Kranke P, Myles P et al. (2007): Society for Ambulatory Anesthesia guidelines for the management of postoperative nausea and vomiting. Anesth Analg 105, $1615-1628$

Gonano C, Kettner SC, Ernstbrunner M, Schebesta K, Chiari A, Marhofer P (2009): Comparison of economical aspects of interscalene brachial plexus blockade and general anaesthesia for arthroscopic shoulder surgery. Br J Anaesth $\underline{103}, 428-33$ 
Gozdemir M, Sert H, Yilmaz N, Kanbak O, Usta B, Demircioglu RI (2007): Remifentanil-propofol in vertebral disk operations: hemodynamics and recovery versus desflurane-n(2)o inhalation anesthesia. Adv Ther 24, 622-31.

Graf BM, Martin E (2001): Periphere Nervenblockaden. Anaesthesist 50, 312-322

Greenberg CP (1995): Practical, cost-effective regional Aanesthesia in ambulatory surgery. J Clin Anesth $\underline{7}, 614-621$

Greenblatt GM, Denson JS (1962): Needle nerve stimulatorlocator: nerve blocks with a new instrument for locating nerves. Anesth and Analg $\underline{41}, 599-602$

Griffin J, Nicholls B (2010): Ultrasound in regional anaesthesia. Anaesthesia $\underline{65}$ (Suppl. 1), 1-12

Grossi P, Calliada S, Braga A, Caldara P, D'Alola A, Coluccla R (1998):

Interscalene brachial plexus block combined with total intravenous anaesthesia and laryngeal mask airway for shoulder surgery. Anaesthesia 53 (Suppl. 2), 1-80

Grundmann U, Risch A, Kleinschmidt S, Klatt R, Larsen R (1998): RemifentanilPropofol-Anästhesie bei Bandscheibenoperationen: ein Vergleich mit einer Desfluran- $\mathrm{N}_{2} \mathrm{O}-$ Inhalationsanästhesie. Anaesthesist $\underline{47}, 102-110$

Guignard B, Bossard AE, Coste C, Sessler DI, Lebrault C, Alfonsi P, Fletcher D, Chauvin M (2000): Acute opioid tolerance. Anesthesiology 93, 409-17

Gupta A (2007): Evidence-based medicine in day surgery. Curr Opin Anaesthesiol 20, 520-525.

Hadzic A, Karaca PE, Hobeika P, Unis G, Dermksian J, Yufa M, Claudio R, Vloka JD, Santos AC, Thys DM (2005) Peripheral nerve blocks result in superior recovery profile compared with general anesthesia in outpatient knee arthroscopy. Anesth Analg 100, 976-981

Heavner JE (2007): Local anesthetics. Curr Opin Anaesthesiol 20, 336-342.

Heller R, Nollert U, Entholzner E (2009): Anästhesie bei ambulanten Patienten. Anaesthesist 58, 421-431

Hempel V (1999): Anästhesie des Plexus brachialis. Anaesthesist 4ㅇ, 341-355

Hogan QH (2008): Pathophysiology of peripheral nerve injury during regional anesthesia. Reg Anesth Pain Med 33, 435-441. 
Hogue CW, Bowdle TA, O’Leary C, Duncalf D, Miguel R, Pitts M, Streisand J, Kirvassilis G, Jamerson B, McNeal S et al. (1996): A multicenter evaluation of total intravenous anesthesia with remifentanil and propofol for elective inpatient surgery. Anesth Analg $\underline{83}, 279-85$

Hong JY, Kang YS, Kil HK (2008): Anaesthesia for day case excisional breast biopsy:propofol-remifentanil compared with sevoflurane-nitrous oxide. Eur J Anaesthesiol 25, 460-467

Horlocker TT, Kufner RP, Bishop AT, Maxson PM, Schroeder DR (1999): The risk of persistent paresthesia is not increased with repeated axillary block. Anesth Analg $\underline{88}, 382-387$

Horn JL, Swide C, Gaebel BA, Cross RL Jr (2007): Comparison of efficiency, recovery profile and perioperative costs of regional anaesthesia vs. general anaesthesia for outpatient upper extremity surgery. Eur J Anaesthesiol 24, 557-559

Hortense A, Perez MV, Gomes do Amaral JL, Martins de Vasconcelos Oshiro AC, Rossetti HB (2010): Interscalene brachial plexus block. Effects on pulmonary function. Rev Bras Anestesiol 60, 130-137

Jeong H, Jeong S, Lim HJ, Lee J, Yoo KY (2012): Cerebral oxygen saturation measured by near-infrared spectroscopy and jugular venous bulb oxygen saturation during arthroscopic shoulder surgery in beach chair position under sevofluranenitrous oxide or propofol-remifentanil anesthesia. Anesthesiology 116, 1047-1056

Juckenhöfel S, Feisel C, Schmitt HJ, Biedler A (1999): TIVA mit Propofol/ Remifentanil oder balancierte Anästhesie mit Sevofluran/Fentanyl bei laparoskopischen Operationen. Anaesthesist 48, 807-812

Kahn RL, Hargett MJ (1999): Beta-adrenergic blockers and vasovagal episodes during shoulder surgery in the sitting position under interscalene block. Anesth Analg $\underline{88}, 378-381$

Kassenärztliche Bundesvereinigung (2006): Vertrag nach § 115 b Abs. 1 SGB V Ambulantes Operieren und stationsersetzende Eingriffe im Krankenhaus - (AOPVertrag). Dtsch Arztebl 103, A2578-A2582

Kempen P, O'Donnell J, Lawler R, Mantha V (2000): Acute respiratory insufficiency during interscalene plexus block. Anesth Analg 90, 4015-4016 
Kinsella SM, Tuckey JP (2001): Perioperative bradycardia and asystole: relationship to vasovagal syncope and the Bezold-Jarisch reflex. Br J Anaesth $\underline{86}$, 859-868

Klein SM, Nielsen KC, Greengrass RA, Warner DS, Martin A, Steele SM (2002): Ambulatory discharge after long-acting peripheral nerve blockade: 2382 blocks with ropivacaine. Anesth Analg $\underline{94}, 65-70$

Kochs E, Adams HA, Spies C: Regionale Anästhesieverfahren. In:

Anästhesiologie; hrsg. v. Wagner KJ, Selenzov V; Georg Thieme Verlag, Stuttgart 2009, 723-790

Kumar A, Battit GE, Froese AB, Long MC (1971): Bilateral cervical and thoracic epidural blockade complicating interscalene brachial plexus block: report of two cases. Anesthesiology $\underline{35}, 650-652$

Kwak HJ, Lee JS, Lee YD, Kim SY, Chang JS (2010): The effect of a sequential compression device on hemodynamics in arthroskopic shoulder surgery using beach-chair position. Arthroscopy 26, 729-733

Lee M, Silverman S, Hansen H, Patel V, Manchikanti L (2011): A comprehensive review of opioid-induced hyperalgesia. Pain Physician 14, 145-161

Liguori GA, Kahn RL, Gordon J, Gordon MA, Urban MK (1998): The use of metoprolol and glycopyrrolate to prevent hypotensive/bradycardic events during shoulder arthroscopy in the sitting position under interscalene block. Anesth Analg $\underline{87}, 1320-1325$

Liu SS, Strodtbeck WM, Richman JM, Wu CL (2005): A comparison of regional versus general anesthesia for ambulatory anesthesia: a meta-analysis of randomized controlled trials. Anesth Analg 101,1634-1642

Lux EA, Stamer U, Meissner W, Moser K, Neugebauer E, Wiebalck A (2008):

Postoperative Schmerztherapie nach ambulanten Operationen. Schmerz 22, 171-175

Lux EA, Stamer U, Meissner W, Wiebalck A (2011): Postoperative Schmerztherapie nach ambulanten Operationen Eine Befragung der behandelnden Anästhesisten. Schmerz 25, 191-194

Matthey PW, Finegan BA, Finucane BT (2004): The public's fears about and perceptions of regional anesthesia. Reg Anesth Pain Med 29, 96-101. 
McCartney CJ, Brull R, Chan VW, Katz J, Abbas S, Graham B, Nova H, Rawson R, Anastakis DJ, von Schroeder H (2004): Early but no long-term benefit of regional compared with general anesthesia for ambulatory hand surgery. Anesthesiology 101, 461-467

Meier G, Bauereis C, Heinrich C (1997): Der interskalenäre Plexuskatheter zur Anästhesie und postoperativen Schmerztherapie - Erfahrungen mit einer modifizierten Technik. Anaesthesist $\underline{46}, 715-719$

Meier G, Bauereis C, Maurer H, Meier T (2001): Interskalenäre Plexusblockade. Anaesthesist 50, 333-341

Möllmann M (2011): Zukunftsorientierte Gestaltung des ambulanten Operierens. Anaesthesist 60, 986-994

Neuburger M, Büttner J (2011): Komplikationen bei peripherer Regionalanästhesie. Anaesthesist 60, 1014-1026

Neuburger M, Rotzinger M, Kaiser H. (2001): Elektrische Nervenstimulation in Abhängigkeit von der benutzten Impulsbreite: Eine quantitative Untersuchung zur Annäherung der Nadelspitze an den Nerven. Anaesthesist 50, 181-186

O'Donnell BD, Iohom G (2008): Regional anesthesia techniques for ambulatory orthopedic surgery. Curr Opin Anaesthesiol 21, 723-728

Ozzeybek D, Oztekin S, Mavioglu O, Karaege G, Ozkardes Sler S, Ozkan M, Canyilmaz M, Elar Z (2003): Comparison of the haemodynamic effects of interscalene block combined with general anaesthesia and interscalene block alone for shoulder surgery. J Int Med Res $\underline{31}$, 428-433

Passannante AN (1996): Spinal anesthesia and permanent neurologic deficit after interscalene block. Anesth Analg $\underline{82}, 873-874$

Ritchie JM, Ritchie B, Greengard P (1965): The active structure of local anesthetics. J Pharmacol Exp Ther 150, 152-159

Ross S, Scarborough CD (1973): Total spinal anesthesia following brachial-plexus block. Anesthesiology $\underline{39}, 458$

Rowbotham DJ, Peacock JE, Jones RM, Speedy M, Sneyd R, Morris RW, Nolan JP, Jolliffe D, Lang G (1998): Comparison of remifentanil in combination with isoflurane or propofol for short-stay surgical procedures. Br J Anaesthesia $\underline{80}$, $752-755$ 
Sala-Blanch X, Lázaro JR, Correa J, Gómez-Fernandez M (1999): Phrenic nerve block caused by interscalene brachial plexus block: effects of digital pressure and a low volume of local anesthetic. Reg Anesth Pain Med 24, 231-235

Schulz-Stübner S (2003): Plexus brachialis Anästhesie und Analgesie. Anaesthesist $\underline{52}, 643-656$

Schuster M, Gottschalk A, Berger J, Standl T (2005): A retrospective comparison of costs for regional and general anesthesia techniques. Anesth Analg 100, 786-794

Schwemmer U, Greim CA, Boehm TD, Papenfuss T, Markus CK, Roewer N, Gohlke F (2004): Perioperative Schmerzbehandlung in der Schulterchirurgie. Schmerz $\underline{18}, 475-480$

Seltzer JL (1977): Hoarseness and Horner's syndrome after interscalene brachial plexus block. Anesth Analg $\underline{56}, 585-586$

Shevade K, Panagopoulos G (1991): A survey of 800 patients' knowledge, attitudes, and concerns regarding anesthesia. Anesth Analg $\underline{73}$, 190-198

Shnaider I, Chung F (2006): Outcomes in day surgery. Curr Opin Anesthesiol 19, 622-629

Statistisches Bundesamt (2010): Krankenhäuser mit ambulanten Operationen und Anzahl von ambulanten Operationen. www.gde-bund.de

Stiglitz Y, Gosselin O, Sedaghatian J, Sirveaux F, Mole D (2011): Pain after shoulder arthroscopy: a prospective study on 231 cases. Orthop Traumatol Surg Res 97, 260-266

Trentman TL, Fassett SL, Thomas jK, Noble BN, Renfree KJ, Hattrup SJ (2011): More hypotension in patients taking antihypertensives preoperatively during shoulder surgery in the beach chair position. Can J Anaesth $\underline{58}$, 993-1000

Trompeter A, Camilleri G, Narang K, Hauf W, Venn R (2010): Analgesia requirements after interscalene block for shoulder arthroscopy: the 5 days following surgery. Arch Orthop Trauma Surg 130, 417-421

Urmey WF, Talts KH, Sharrock NE (1991): One hundred percent incidence of hemidiaphragmatic paresis associated with interscalene brachial plexus anesthesia as diagnosed by ultrasonography. Anesth Analg $\underline{72}, 498-503$ 
Vester-Andersen T, Christiansen C, Hansen A, Sørensen M, Meisler C (1981): Interscalene brachial plexus block: area of analgesia, complications and blood concentrations of local anesthetics. Acta Anaesthesiol Scand 25, 81-84.

Walton JS, Folk JF, Friedman RJ, Dorman BH (2000): Complete brachial plexus palsy after total shoulder arthroplasty done with interscalene block anesthesia. Reg Anesth Pain Med 25, 318-321

Webster F, Bremner S, McCartney CJ (2011): Patient experiences as knowledge for the evidence base: a qualitative approach to understanding patient experiences regarding the use of regional anesthesia for hip and knee arthroplasty. Reg Anesth Pain Med $\underline{36}, 461-465$

White PF, Eng M (2007): Fast-track anesthetic techniques for ambulatory surgery. Curr Opin Anaesthesiol 20, 545-557

Williams BA, Motolenich P, Kentor ML (2005): Hospital facilities and resource management: economic impact of a high-volume regional anesthesia program for outpatients. Int Anesthesiol Clin $\underline{43}, 43-51$.

Winnie AP (1970): Interscalene brachial plexus block. Anesth Analg $\underline{49}, 455-466$

Woolf CJ, Chong MS (1993): Preemptive analgesia-treating postoperative pain by preventing the establishment of central sensitization. Anesth Analg 77, 362-379

Yadeau JT, Liu SS, Bang H, Shaw PM, Wilfred SE, Shetty T, Gordon M (2011): Cerebral oximetry desaturation during shoulder surgery performed in a sitting position under regional anesthesia. Can J Anaesth $\underline{58}$, 986-992

Zielmann S, Kazmaier S, Schnüll S, Weyland A (1997): S-(+)-Ketamin und Kreislauf. Anaesthesist 46 (Suppl 1), S43-S46

Zink W, Graf BM (2003): Toxikologie der Lokalanästhetika. Patho-mechanismen Klinik - Therapie. Anaesthesist 52, 1102-1123 


\section{Publikation:}

Janssen H, von Stosch R, Pöschl R, Büttner B, Bauer M, Hinz JM, Bergmann I (2014): Blood pressure response to combined general anaesthesia/interscalene brachial plexus block for outpatient shoulder arthroscopy. BMC Anaesthesiology 14,50

\section{Danksagung:}

Ich möchte mich herzlich bei Dr. I. Bergmann und Prof. Dr. J. Hinz für Ihre Unterstützung beim Verfassen dieser Arbeit bedanken!

Mein besonderer Dank gilt außerdem Dr. R. Pöschl und Dr. R. von Stosch für die Zusammenarbeit beim Erfassen der Daten. 


\section{Lebenslauf}

Mein Name ist Hauke Onko Janssen, geboren am 22.02.1980 in Göttingen. Ich wohne zusammen mit meiner Frau Iryna Janssen, mit der ich seit dem 06.04.2013 verheiratet bin, in der Herzberger Landstraße 36 in 37085 Göttingen.

Nach meiner Grundschulzeit von 1986 bis 1990 an der Lohberg-Schule in Göttingen besuchte ich die Georg-Christoph-Lichtenberg-Gesamtschule in Göttingen und schloss am 19.05.1999 mit der allgemeinen Hochschulreife ab. Während meiner Schulzeit habe ich mich vor allem für naturwissenschaftliche Fächer interessiert und in der Sekundarstufe II die Leistungskurse Biologie und Chemie belegt. Mein Interesse für Medizin begann 1996 mit meiner ehrenamtlichen Mitgliedschaft in der Johanniter-Unfall-Hilfe e.V.. Dort habe ich mich in den Bereichen Jugendarbeit und Sanitätsdienst engagiert. Ich habe dort neben einer JugendgruppenleiterGrundausbildung auch eine Ausbildung zum Rettungssanitäter begonnen, die ich am 11.08.2001 erfolgreich abschloss. Zeitweise war ich im Bereich des Katastrophenschutzes aktiv und habe am 03.02.2000 erfolgreich eine Ausbildung zum Gruppenführer im Bereich Sanitätsdienst/Betreuungsdienst abgeschlossen.

Nach meiner Schulzeit habe ich vom 02.08.1999 bis 30.06.2000 meinen Zivildienst im Krankenhaus Neu-Bethlehem in Göttingen in der Krankenpflege auf einer allgemeinchirurgischen Station abgeleistet, bevor ich dann im Oktober 2000 mein Studium der Humanmedizin an der Georg-August-Universität in Göttingen begann. Am 19.09.2003 habe ich die ärztliche Vorprüfung abgelegt und am 29.05.2008 meine Approbation als Arzt erhalten. Während meines Studiums habe ich Famulaturen im Bereich Unfallchirurgie im Marien-Krankenhaus in Papenburg, in der ambulanten Anästhesie bei Dr. Fritz Jähn in Göttingen, im Bereich Innere Medizin/Kardiologie im Krankenhaus Neu-Bethlehem in Göttingen und im Bereich Innere Medizin und Pädiatrie im Wukro-District-Hospital in Wukro/ Äthiopien abgeleistet. Außerdem habe ich von Oktober 2001 bis März 2008 bei Dr. Fritz Jähn in Göttingen und von September 2005 bis März 2008 bei Dr. Rupert 
Pöschl in Baunatal als studentische Hilfskraft in der ambulanten Anästhesie gearbeitet. Mein Praktisches Jahr von August 2006 bis Juli 2007 habe ich an der Universitätsmedizin Göttingen in den Abteilungen Thorax-Herz-Gefäßchirurgie, Anästhesiologie und Innere Medizin/Gastroenterologie absolviert.

Seit dem 01.08.2008 bin ich an der Universitätsmedizin Göttingen im Zentrum für Anästhesiologie, Rettungs- und Intensivmedizin als Arzt beschäftigt. Am 25.02.2013 habe ich die Zusatzbezeichnung Notfallmedizin erhalten und am 24.09.2013 erfolgreich meine Prüfung zum Facharzt für Anästhesiologie abgelegt. Im Rahmen eines Kooperationsprojektes zwischen der Universitätsmedizin Göttingen und des St. Martin de Porres Hospital in Eikwe/Ghana bekam ich die Möglichkeit, vom 01.11.2013 bis zum 31.10.2014 einen stipendiengeförderten Forschungs- und Qualifizierungsaufenthalt in Eikwe/Ghana zu absolvieren. Im St. Martin de Porres Hospital habe ich im Bereich Anästhesiologie gearbeitet und Fortbildungen des dortigen Personals zum Thema „Innerklinische Notfallmedizin“ durchgeführt. Aufgrund des Ebola-Ausbruchs in West-Afrika Anfang des Jahres 2014 habe ich außerdem Ausbildungen zum Thema „Infektionsschutz“ durchgeführt und das Krankenhaus bei Vorbereitungen auf einen Ebola-Fall unterstützt.

Zur Zeit arbeite ich in der Intensivmedizin der Klinik für Anästhesiologie der Universitätsmedizin Göttingen, um die Zusatzbezeichnung „Spezielle Anästhesiologische Intensivmedizin“ zu erlangen. 